\title{
1 Molecular insights into the pathways underlying naked mole-rat eusociality
}

3 Eskeatnaf Mulugeta ${ }^{1,2^{*}}$, Lucile Marion-Poll ${ }^{1,2}$, David Gentien ${ }^{1,2}$, Stefanie B. Ganswindt ${ }^{3}$, André

4 Ganswindt $^{3}$, Nigel C. Bennett ${ }^{4}$, Elizabeth H. Blackburn ${ }^{5}$, Chris G. Faulkes ${ }^{6^{*}}$, Edith Heard ${ }^{1,2}$

6 Author Affiliations

71 Institut Curie, PSL Research University, CNRS UMR3215, INSERM U934, Mammalian

8 Developmental Epigenetics group, F-75005 Paris, France

92 Sorbonne Universités, UPMC Univ Paris 6, F-75005 Paris, France

103 Faculty of Veterinary Science, University of Pretoria, Onderstepoort 0110, South Africa

114 Department of Zoology and Entomology, University of Pretoria, Pretoria 0002, South Africa

125 Salk Institute for Biological Studies, California, USA

136 School of Biological and Chemical Sciences, Queen Mary University of London, England

$14 * \quad$ Corresponding authors

15

16 Correspondence to: Eskeatnaf Mulugeta (eskeatnaf.mulugeta@curie.fror sskeww@gmail.com) Chris G. Faulkes (c.g.faulkes@qmul.ac.uk)

\section{Keywords:}

22 Naked mole-rat, Heterocephalus, Bathyergidae, Eusociality, Aromatase, Dopaminergic pathways,

23 Prolactin, Hyperprolactinemia, Reproductive suppression 


\section{Abstract}

\section{Background:}

28 Eusociality is the highest level of social organization and naked mole-rats (NMR)s are amongst the few 29 mammals showing this unique social behavior; nevertheless, little is known about the molecular 30 mechanisms underlying the eusociality of NMRs.

\section{Results:}

32 Gene expression profiling of NMR brain and gonads (ovary and testis), from animals belonging to 33 different reproductive castes, revealed robust gene expression differences between reproductive and non34 reproductive members of NMR colonies. In the brain, dopaminergic pathways appear to be potential 35 players in NMR eusocial behaviour. Breeding animals (queens and breeding males) showed increased 36 expression of genes involved in dopamine metabolism. Using immunohistochemistry, we notably found 37 these differences to be in dopaminergic hypothalamic areas, which provide inhibitory control over the secretion of prolactin, amongst other regions. Furthermore, plasma prolactin concentrations were elevated in many non-breeders (of both sexes), often reaching levels exceeding that of pregnant or lactating

40 queens, suggesting a role for hyperprolactinaemia in socially-induced reproductive suppression. We also

41 found that the ovaries of non-breeding females are arrested at pre-pubertal stage. They contained fewer 42 supporting stromal cells compared to queens, and had very low expression of the aromatase gene 43 Cyp19A1 (a key enzyme in estrogen synthesis) compared to non-breeding females. In the testes, genes 44 involved in post meiosis spermatogenesis and sperm maturation (Prm1, Prm2, Odf3 and Akap4) were 45 highly expressed in breeding males compared to non-breeders, explaining the low sperm number and 46 impaired sperm motility characteristic of non-breeding males.

\section{Conclusions:}

48 Our study suggests that extreme reproductive skew, one of the defining features of eusociality, is 49 associated with changes in expression of key components of dopamine pathways, which could lead to 50 hypogonadism and a lifetime of socially-induced sterility for most NMRs. 


\section{Background}

53 Eusociality is the highest level of social organization and is perceived as one of the major transitions in

54 the evolution of life $[1,2]$. The original definition, derived from studies of social insects, requires three

55 criteria: a reproductive division of labour, overlapping generations, and cooperative care of young [3, 4],

56 although it has been argued that physically distinct morphological castes should also be present [5]. This

57 extraordinary cooperative way of life is famously observed in invertebrates, such as the social

58 hymenoptera, but also other diverse groups such as crustaceans, and more recently, some mammals [6-9].

59 The importance of eusociality as a strategy is exemplified by the fact that eusocial species may constitute

$6075 \%$ of the insect biomass in some ecosystems $[10,11]$. The taxonomic diversity of eusociality has led to

61 much debate about its definition, especially among mammals (for review see [12]), but it is generally

62 accepted that among the African mole-rats (Family: Bathyergidae), the naked mole-rat (Heterocephalus

63 glaber) and Damaraland mole-rat (Fukomys damarensis) have independently evolved eusociality [8, 13,

$6414]$.

65

66 NMRs live entirely underground in the arid regions of East Africa in colonies that may contain up to 300

67 individuals [15]. Most colonies of NMRs have only one breeding female (the queen), who mates with one

68 to three selected males, and the rest of the colony (both sexes) are reproductively suppressed $[8,16]$. The

69 reproductive status of the queen and the breeding males may be stable for many years - NMRs can live up

70 to 32 years in captivity [17]. The queen is dominant in the colony social hierarchy, and evidence suggests

71 that she exerts a dominant control mechanism of reproductive suppression over the non-breeders of both

72 sexes, and possibly also the breeding male [18-21]. The remaining members of the colony of both sexes

73 are morphologically very similar (externally) and do not exhibit sexual behavior, but perform tasks that

74 are essential for the survival and wellbeing of the colony: foraging, colony defense, maintenance of the

75 tunnel system, and care of the young $[8,16]$. It has been estimated that more than $99 \%$ of non-breeders

76 never reproduce [16]. However, the extreme socially-induced suppression of reproduction is reversible 
77 upon removal of the suppressing cues: if the queen or breeding males die or are removed from the colony,

78 or if non-breeding animals are housed singly or in pairs [18, 20-23].

80 Non-breeding females remain at pre-pubertal anovulatory state despite attaining adult body size, with

81 small uteri and ovaries, and reduced plasma luteinizing hormone (LH) concentrations that is reflected in

82 lower plasma and urinary progesterone levels $[18,19,24]$. Upon removal of the queen, and often after

83 fighting and competition among non-breeding females, one non-breeding female attains breeding status,

84 and undergoes anatomical, behavioral, and endocrine changes, including elongation of the body,

85 perforation of the vagina, increased dominance and aggression, and activation of the ovaries and

86 ovulation, with resulting increased urinary and plasma progesterone [22, 24-26]. The degree of

87 suppression in non-breeding males is less pronounced than the suppression in non-breeding females in

88 that gametes are produced. Non-breeding males have lower urinary testosterone levels, plasma luteinizing

89 hormone (LH), and lower reproductive tract to body mass ratio compared to breeders [20, 27].

90 Nevertheless, non-breeding males are able to undergo spermatogenesis and mature spermatozoa are

91 detected in their epididymis and vas deferens, although their number is lower than in breeding males and

92 the majority are non-motile [28]. This indicates that the low concentration of testosterone and LH in non-

93 breeding males is sufficient to support the spermatogenetic cycle, but not the final stages of maturation

94 steps (pituitary FSH may also be inhibited, but to date has not been measured in NMRs). As with females,

95 suppressive effects are reversible upon withdrawal of non-breeding males from their parent colonies and

96 housing singly or pairing with a female - increases in concentrations of urinary testosterone levels and

97 plasma luteinizing hormone (LH) occur rapidly [20].

98

99 The detailed mechanism underlying this reproductive suppression remains elusive. The central role of 100 hypothalamic gonadotrophin releasing hormone $(\mathrm{GnRH})$ in integrating environmental cues is well 101 established [29-31], while reproductive development and puberty may be dependent on another 102 hypothalamic peptide, kisspeptin (acting via GnRH) [32]. In NMRs, a lack of priming of the pituitary 
gland by impaired release of hypothalamic GnRH may be a key component in reproductive suppression not differ between breeding and non-breeding NMRs within or between the sexes [33]. However, breeding females have greater numbers of kisspeptin cell bodies in key areas of the hypothalamus, suggesting that emergence from a socially-induced hypogonadotrophic/prepubertal state in female NMRs may involve kisspeptin-related pathways. Recently, elevated levels of the RF amide related protein 3 (RFRP-3 or GnIH) in the brain of non-breeders have been implicated as a component in the suppression of reproduction in NMRs, through the inhibition of GnRH secretion [34]. It is currently unknown how behavioural and other sensory cues (such as signature odours and vocalisations) mediate the extreme social suppression of reproduction in NMRs.

In recent years, gene expression profiling of several eusocial insects have provided insight into the molecular mechanisms and evolutionary paths to eusociality in insects [35-42]. However, although genomic and transcriptomic analyses of NMRs and other mole-rats have also been published, these have

117 focused on individual animals or cross species comparisons [43-45]. So far no comprehensive comparative analysis within and among sexes and reproductive castes of NMRs has been reported. Here we use RNA-sequencing (RNA-seq) to undertake the extensive transcriptome profiling of breeding and non-breeding NMR brains and gonads (ovary and testis). This has revealed striking gene expression

121 differences that point to the possible mechanisms underlying eusociality in a mammal, and extreme 122 socially-induced reproductive suppression.

\section{Results}

\section{Changes in the dopaminergic system are strongly associated with NMR reproductive status}

127 In order to determine the origin of the behavioral and reproductive differences between NMR social 128 classes and elucidate the molecular pathways that contribute to these differences, we performed gene 
expression profiling using very high coverage RNA-Seq on whole brains of several animals that constitute both sexes and different reproductive classes (Additional file 1A: animals used and Additional file $1 B$ : RNA-seq information). We first explored the global expression levels, and based on principal component analysis (PCA), the first principal components clearly captured the reproductive caste differences: with the queens and other castes distinctly clustering in their respective groups. Similarly, using a hierarchical clustering technique, non-pregnant NMR queens (Qs) cluster separately to the rest of the colony members (Supplementary Figure 1A,B). The rest of the colony members - non-breeding females (NBFs), non-breeding males (NBMs), breeding males (BMs), and a pregnant $\mathrm{Q}$ clustered as another group, with the majority of NBFs and NBMs clustering as one group, independent of sex (Supplementary Figure 1A,B). In order to define the gene expression differences between brains, we performed differential expression analysis (see Methods) and identified several genes that show significant gene expression differences $(\mathrm{FDR}<0.05$ Benjamini-Hochberg multiple testing correction; a $\log 2$ fold change $>1$; and $>1$ CPM, counts per million) between breeding and non-breeding animals

144 breeding animals compared to non-breeding animals, and with minor differences between subordinate animals (Figure 1A,B).

147 To elucidate the nature of these gene expression differences in more detail we examined each group in 148 pairwise comparisons. We first focused on differences between Qs $(n=2)$ and NBFs $(n=3)$. Based on a 149 global gene expression comparison, the Qs and NBFs clearly cluster as two distinct groups, suggesting 150 the clear gene expression difference in the brain of Qs and NBFs (Supplementary Figure 2A,B). In total, 151854 genes were differentially expressed (389 higher expression in the Qs and, 465 lower expression in 152 Qs) (Figure 1A,B see Qs vs NBFs, Supplementary Figure 2C, Additional file 2). These DEGs are enriched 153 for pathway and biological process terms that include synaptic signaling, dopaminergic synapse, positive 154 regulation of transport, and neurotransmitter receptor activity (Supplementary Figure 2D,E,F, G, and 
Additional files 3, 4, 5). Interestingly, genes related to dopamine were particularly enriched in the DEGs.

Dopamine is a catecholamine (a monoamine compound derived from the amino acid tyrosine) and has a neurotransmitter function. Several key-genes, such as the tyrosine hydroxylase $(T h)$ gene (the ratelimiting enzyme in the synthesis of catecholamines), the vesicular monoamine transporter (Vmat2 or Slc18a2) which transports monoamine neurotransmitters into the vesicles to be released at the synapse, and the dopamine transporter (Dat or Slc6a3) responsible for the reuptake of dopamine were found to be highly expressed in the Qs compared to the NBFs (Additional file 2). Th and Dat were both in the top 10 of our DEG list, suggesting higher dopamine production in the Qs brain. In addition, the most abundant dopamine receptors Drdla, Drd2 and Drd3 were all down-regulated in the Qs, while the Drd5 receptor gene was up-regulated (Additional file 2). Genes related to the other catecholamines adrenalin and noradrenalin (dopamine beta-hydroxylase Dbh, phenylethanolamine N-methyltransferase Pnmt, and the different adrenergic receptors alpha and beta) were not differentially expressed between Qs and NBFs, indicating that the differences are specific to the dopaminergic system.

Next, we looked at the gene expression differences between BMs and NBMs. As breeding status of male NMRs can be difficult to determine [28], we used two criteria to define their reproductive status in the colony: 1) long-term observational information, and 2) testis to body mass ratio (Supplementary Figure $3 A$ ). On PCA and hierarchical clustering (using global gene expression), unlike the distinction between

173 Qs and NBFs, BMs and NBMs do not fully resolve into separate clusters (Supplementary Figure 1B,

174 Supplementary Figure 3B,C). For animals that fulfill the two criteria above (3 BMs and 3 NBMs, 175 excluding one NBM whose status was uncertain), we performed differential gene expression analysis and 176 identified 193 genes (with 152 showing higher expression in BMs and 42 lower expression in BMs; 177 Figure $1 A$ B see Qs vs BMs, Supplementary Figure 3 D, Additional file 6). These DEGs are enriched for 178 pathways and biological processes that are involved in nitrogen compound transport, regulation of 179 nervous system development, synaptic signaling, dopaminergic neuron differentiation, behavior 180 (Supplementary Figure 3E,F, G,H and Additional files 7, 8, 9). Similarly to the comparison between Qs vs 
NBFs, we also found Th and Dat to be amongst the most highly expressed genes in BMs compared to NBMs (Additional file 6), suggesting that similar mechanisms, involving the dopaminergic pathway, may account for the behavioral and reproductive differences observed between BMs and NBMs.

Breeding animals (Qs and BMs) are the most dominant and aggressive in the NMR colony. These animals, Qs and BMs, clearly cluster into independent groups (Supplementary Figure 1A,B, Supplementary Figure 4A), and also show the highest number of DEGs (Figure 1A, B, see Qs vs BMs). In total 1246 genes show significant expression difference between Qs and BMs (444 higher expression in Qs and 802 lower expression in Qs; Figure 1A,B, Supplementary Figure 4C, and Additional file 10). These DEGs are enriched for pathways and biological processes that are involved in protein targeting to membrane, mRNA metabolic process, cellular macromolecule catabolic process, regulation of cellular response to stress and related pathways (Supplementary Figure 4D,E,F,G, and Additional file 11, 12, 13). agreement with previous observations showing the absence of behavioral differences (including sexual characteristics), and sexual differentiation (including similarity in external genitals) between NBMs and NBFs [46]. 
we focused on genes that are found in the comparison between Qs vs NBFs and Qs vs NBMs (Figure 1C,

Supplementary Figure $6 D, E$, Additional file 16). Around half of the genes that were differentially

expressed between Qs vs NBFs are shared with Qs vs NBMs (Figure 1C). These Q genes are enriched in

pathways and biological process terms that are involved in behavior, synaptic signaling, multi-organism

behavior, dopaminergic synapse, neurotransmitter transport, dopamine binding, reproductive behavior

and other related terms (Figure 1D,E, Additional file 17, 18, 19). We performed a similar analysis to

213 define genes that are unique to the BMs by taking genes that are common in the comparison between

214 BMs vs NBFs (Additional file 20) and BMs vs NMBs (Figure 1A,B, Additional file 21). In general, while

215 we found fewer such BM genes compared to Q genes (Supplementary Figure $6 F, G, H$ ), these genes are

216 involved in similar pathways as the Q genes. Similar to the Q genes, BM genes are also enriched for

217 visual perception, nitrogen compound transport, behavior, neuron projection morphogenesis,

218 dopaminergic synapse (Additional file 22, 23, 24). In addition, similarly to the Q gene list, Th and Dat

219 were also present in the BM gene list (unique in BMs vs NBMs and BMs vs NBFs). These results (Q

220 genes and BM genes) highlight the possible role of dopamine pathway in the NMR colony social

221 behavior and reproductive suppression.

223 Our detailed analysis of NMR brain RNA-seq has identified the dopamine system as a probable key

224 player in the breeding status differences. There are several cell groups in the brain which are potentially dopaminergic (named A8 to A16), linked to different functions [47]. In order to independently confirm the differences in the dopamine system between reproductive castes and to explore their localization, we collected brains from a different batch of animals (Additional file 1A). Even though the Qs were bigger in size compared to the other animals (56 $\pm 16 \mathrm{~g}$ versus $34 \pm 3 \mathrm{~g}$ ), all the brains had similar sizes with no obvious neuromorphological differences across the castes. We performed immunostaining against Tyrosine hydroxylase (TH) on several sections along the rostrocaudal axis (Figure 2A). The most caudal sections contained the A9 (substantia nigra compacta) and A10 (ventral tegmental area), nuclei that comprise the vast majority of dopaminergic neurons. Their respective main targets, the dorsal striatum 
and nucleus accumbens, can be seen in the most rostral sections. In addition, we looked at the staining in

234 the hypothalamic nuclei A12, A13 and A14. We observed that across the whole brain, the intensity of the

235 TH staining tended to be higher in breeders than non-breeders, though with inter-individual variability.

236 Strikingly, there was a consistent staining of the hippocampus (and to a lesser extent of the cortex)

237 specifically in all NMR breeders, but absent in non-breeders (Figure 2B), indicating catecholaminergic

238 innervation of this region. To our knowledge, such a strong hippocampal staining has never been reported

239 in mice or rats. Importantly, breeders showed significantly more TH expression in periventricular

240 hypothalamic nuclei A12 and A14 (Figure 2C), which are precisely the two dopaminergic cell groups

241 known to inhibit prolactin (PRL) secretion, a peptide hormone related to lactation and reproduction [48].

243 To investigate our prediction that elevated prolactin (hyperprolactinemia) may be a component in the

244 suppression of reproduction in non-breeding NMRs, we measured plasma PRL in breeding and non-

245 breeding animals of both sexes in samples taken across thirteen colonies (Figure $1 F)$. We found that most

246 samples from non-breeders had detectable concentrations of PRL, and these often reached very high

247 levels: NBF (mean \pm SEM) $32.64 \pm 6.13 \mathrm{ng} / \mathrm{ml}$; $\mathrm{n}=44$; range, 0.03-173.57 ng/ml; NBM $36.77 \pm 9.81$

$248 \mathrm{ng} / \mathrm{ml} ; \mathrm{n}=49 ;$ range, 0.03-330.30 ng/ml. Among breeders, queens had the expected variance in plasma

249 PRL concentrations as part of normal ovarian cyclicity, pregnancy and lactation: $33.02 \pm 12.94 \mathrm{ng} / \mathrm{ml}$;

$250 \mathrm{n}=12$; range, $3.60-160.80 \mathrm{ng} / \mathrm{ml}$. Two values were obtained from lactating queens, $21.14 \mathrm{ng} / \mathrm{ml}$ (23 days

251 post-partum, at the end of the period of lactation) and $160.80 \mathrm{ng} / \mathrm{ml}$ (seven days post-partum), the latter

252 being the highest concentration recorded among the breeding female samples. Although the sample size

253 was low due to the difficulties of identifying them, breeding males had low plasma PRL concentrations,

254 as predicted from our model of suppression: $15.91 \pm 6.21 \mathrm{ng} / \mathrm{ml} ; \mathrm{n}=7$; range, $3.92-47.92 \mathrm{ng} / \mathrm{ml}$.

256 In conclusion, this comparison of gene expression profiles in NMR brains uncovers differences that

257 follow social status rather than sex, with the Q having a unique gene expression pattern compared to the 
rest of the colony. Furthermore, we identify dopaminergic pathways as potential key players in NMR eusocial behavior.

\section{Non-breeding female NMRs have pre-pubertal ovary and lack the production of ovarian estrogen}

To characterize the origin of the differential reproductive behavior of Qs and NBFs, we next focused on gene expression differences between their ovaries. Mammalian oogenesis is a highly regulated process that involves several hormonal and molecular pathways. Oogenesis starts in the fetal ovaries with the development of oogonia from primordial germ cells (PGCs). Each oogonium advances until the first stages of meiosis (meiosis I) and become arrested at the prophase stage of meiosis I, forming primary oocytes $[49,50]$. After puberty, a few primary oocytes are recruited during each ovarian cycle, and only one oocyte matures to be ovulated. The maturation process involves the completion of meiosis I, and generation of secondary oocytes that are again arrested at the metaphase II stage (meiosis II). The second puberty, oocytes in meiosis II into tertiary and pre-ovulating Graafian follicle [50]. The ovaries of NMR NBFs are reported to be at pre-pubertal stage, with mainly primordial follicles, and may be a few secondary or tertiary follicles [51].

Based on PCA and hierarchical clustering, Qs $(n=3)$ and NBFs $(n=3)$ ovaries clearly group by status and cluster separately (Figure 3A,B and Supplementary Figure 7A), supporting previous findings reporting distinct anatomical, endocrine, and physiological differences between the ovaries of Q and NBFs [18, 19,

282 24]. Irrespective of pregnancy or age, Q ovaries group and cluster together separately from NBFs.

283 Differential expression analysis between the Qs and NBFs (excluding the pregnant Q ovary), specifically 
284 identified 1708 genes, with more genes showing significantly lower expression in the Qs compared to

285 NBFs (1175 down regulated genes, lower expression in Qs versus 534 up regulated genes higher

286 expression in Qs compared to NBFs; Figure 3C, Supplementary Figure 7B, Additional file 25). DEGs

287 were globally enriched for biological processes and pathways that are related to regulation of nervous

288 system development, response to lipid, meiotic nuclear division, gland development, negative regulation

289 of developmental process, DNA methylation involved in gamete generation and other related terms

290 (Figure 3D, Supplementary Figure 7C,D,E, Additional file 26A, 27A, 28A). Taking into account the

291 direction of expression changes in Qs versus NBFs, we found that down-regulated genes were enriched

292 for ontology terms related to gamete generation, central nervous system development, developmental

293 process involved in reproduction, meiotic cell cycle process, DNA methylation involved in gamete

294 generation and other related terms (Supplementary Figure 8A,B,C,D, Additional file 26B, 27B, 28B). In

295 contrast, up-regulated genes are mainly enriched for inflammatory response, allograft rejection, leukocyte

296 differentiation, positive regulation of cell differentiation, response to lipid, regulation of anatomical

297 structure morphogenesis, regulation of hematopoiesis, cell adhesion and related pathways (Supplementary

298 Figure 9A,B,C,D, Additional file 26C, 27C, 28C). These results likely reflect the different cellular

299 composition of $\mathrm{Q}$ and NBF ovaries: NBF ovaries are immature and mainly contain primordial and

300 primary follicles (Supplementary Figure 10) while Q ovaries develop abundant stromal cells to support

301 oogenesis/folliculogenesis (Supplementary Figure 10).

303 To investigate ovarian differences in more detail, we first compared our data with available

304 transcriptomes of mouse oocytes at different stages of development [52]. We selected two clusters of

305 genes: a first group that show a decline in expression from primary to small antral follicle stages [52] and

306 the other group where expression increased at small and large antral [52] (753 genes in total in the two

307 clusters). However, we did not observe differences in the expression levels of these genes between Q and

308 NBF ovaries (Supplementary Figure 11A). This could be due to the fact that, in NMRs, only a few

309 follicles progress to the large antral stage at each reproductive cycle, and thus the difference cannot be 
quantified from whole ovary expression data. As NBF ovaries have been reported to be in an immature state $[18,19,24]$, we next integrated available transcriptomes of whole ovaries from pre-puberal and adult mice [53]. Surprisingly, Q and NBF ovaries did not significantly differ in the expression levels of genes that are differentially expressed between pre-pubertal and adult mouse ovaries (Supplementary Figure 11B). However, by focusing on a selection of oocyte-specific genes (Zp1, Zp2, Zp3, Figla, Nobox, Sohlh1, Gja4, Pou5f1, Oosp1, H1foo, Lhx8, Sohlh2, Kit, Kitl, Nlrp14, Bmp15), we observed a consistent increase in NBF compared to Q ovaries (Supplementary Figure 11C). This is in agreement with our histological assessment (Supplementary Figure 10): Q ovaries have more stromal, somatic cells than NBF ovaries, which are enriched in primary oocytes. Upon further examination of the NMR ovary RNA-seq data, in agreement with the enrichment of hormone-producing stromal cells in Q ovaries, we found that the main difference between ovaries of the Qs and NBFs was in the ability to synthesize estrogen. More specifically, among the top-10 list of DEGs, we found that Cyp19A1 (Aromatase) - a key enzyme in the synthesis of estrogen- was highly over-expressed in Q compared to NBF ovaries (4-fold change in $\log 2$ scale with almost no expression in NBF; Supplementary Figure 11D). Aromatase is important for transformation of androstenedione to estrone and testosterone to estradiol $[54,55]$. In addition to

As a whole, our RNA-seq analysis suggests that Q ovaries specifically express genes that are required for estrogen production, and thereby undergoes oogenesis/folliculogenesis, ovulation and other sexual differentiation processes. In contrast, NBFs seem to have functioning ovaries that are arrested at a prepubertal stage, and do not have the ability to produce estrogen, explaining their failure to ovulate and subsequent reproductive incompetency. 
We next wanted to investigate the nature of the gonadal differences between breeding versus non-

throughout life. A pool of spermatogonial stem cells undergo mitotic divisions to produce spermatocytes, that will undergo meiotic divisions to produce haploid spermatids, which will then undergo further maturation to become mature spermatozoa [56-58]. This process of spermatogenesis occurs in seminiferous tubules supported by Sertoli cells and Leydig cells, and coordinated by endocrine signals.

344 Sertoli cells nourish and provide structural support for the developing sperm cells, upon activation by 345 follicle-stimulating hormone (FSH) that is secreted by the pituitary gland, which is in turn under the control of the GnRH secretion from the hypothalamus [58, 59]. Leydig cells that are located outside the seminiferous tubules produce and secrete testosterone and other androgens that are important for spermatogenesis, secondary sexual characteristics, development, and testis volume. Leydig cell function is modulated by the pituitary gonadotrophin luteinizing hormone $(\mathrm{LH})[58,59]$.

All male NMRs, independently of their breeding status, have been reported to undergo spermatogenesis $[19,26,27]$; however, sperm numbers and motility vary between BMs and NBMs [26, 27]. In order to investigate the molecular pathways that contribute to these differences in spermatogenesis between BMs and NBMs, we analyzed the gene expression profile of whole testes from BMs and NBMs, using the two criteria described above in the brain analysis section to define their status (long-term observational information and testis to body mass ratio). Based on PCA and hierarchal clustering, unlike the clear separation observed for the ovaries of Qs and NBFs, NBM testes do not show a distinct clustering and grouping based on breeding status, with BM3 clustering with non-breeding animals (Figure 4A,B,

359 Supplementary Figure 12A). Nevertheless, differential expression analysis identified 780 genes that 360 showed significant changes between BMs and NBMs (522 up-regulated and 258 down-regulated genes in 
BMs compared to NBMs; Figure 4C, Supplementary Figure 12B, Additional file 29). Enrichment analysis for biological processes, molecular functions, and pathways did not highlight obvious enrichment of spermatogenesis genes, but rather for terms such as: regulation of anatomical structure morphogenesis, response to steroid hormone, and embryo development (Figure 4F, Supplementary Figure 12C,D,E Additional file 30A, 31A, 32A). By focusing on DEGs that show lower expression in breeding compared to non-breeding testes, we found enrichment for terms that are related to positive regulation of cellsubstrate adhesion, positive regulation of cell-substrate adhesion, processes regulation of histone acetylation (Supplementary Figure 13A,B,C,D, Additional file 30B, 31B, 32B). However, interestingly, for DEGs that are up-regulated in the testes of BMs we found enrichment of terms related to regulation of anatomical structure morphogenesis, response to steroid hormone, and multicellular organism reproduction (Supplementary Figure 14A,B,C,D, Additional file 30C, 31C, 32C). In particular, this last ontology category (GO:0032504) contains a number of genes (45 genes, Additional file 30D) involved in key biological processes related to male reproduction, such as meiotic cell cycle, male genitalia development, gamete generation, spermatogenesis, and sperm motility (Lhcgr, Prm1, Prm2, Akap4, Odf3, Akap4, Wfdc2, Fdc2, Txnrd2, Txnrd3, Spata6, Spata22, Rara, Nr2c2, Hsf2bp, Cylc2, Cylc1, Celef3, Ccin, Alkbh5, Adam29, Atp2b4; Additional files 30D). From this list of 45 genes, Lhcgr encodes the receptor for both LH and choriogonadotropin [60, 61]. Binding of LH to its receptor stimulates testosterone production by Leydig cells to promote extra-gonadal differentiation and maturation [62,63]. Prm1 and Prm2 genes encode Protamine proteins that replace histones in later stages of spermatogenesis (sperm elongation) to allow denser packing of DNA [64-66]. In mouse models, abnormal expression of Prm1 and Prm2 causes a decrease in spermatozoa number, abnormal spermatozoa morphology and motility, damaged spermatozoa chromatin, and infertility [67-73]. Odf3 is transcribed more specifically in spermatids, and is suggested to provide the elastic structure of sperm protecting from damage during epididymal transport $[74,75]$. Akap4 is transcribed only in the post-meiotic phase of spermatogenesis and is a cytoskeletal structure present in the principal piece of the sperm flagellum [76]. Targeted disruption of Akap4 results in abnormal flagella, and hence motility of spermatozoa [76, 77]. 
To further document what stage of spermatogenesis might be interrupted in NBMs, we imported the list of spermatogenesis-related gene clusters that show specific expression at several different stages of mouse spermatogenesis (mitotic, meiotic, post-meiotic, and somatic clusters,) from the Germonline database (Additional file 30) [78]. DEGs belonging to all four clusters tended to show higher expression in BMs than in NBMs, but this difference was more pronounced for post-meiotic genes (Figure 4D). More specifically, 10\% (55 out of the 522) of up-regulated DEGs in BM belonged to the post-meiotic cluster, while only $2 \%$ of down-regulated DEGs in BM (5 out of 258) belonged to this category. This suggests that the main difference in BM and NBM spermatogenesis is related to genes that are important in post-meiotic stages. In addition, we plotted the expression level of all genes that belong to these four clusters. Genes that belong to both the meiotic and post-meiotic stages of spermatogenesis showed significant up-regulation in BMs compared to NBMs (p-value 0.01 and 0.002 respectively, Wilcoxon rank sum test; Figure $4 E$ ), but this trend was more pronounced for the post-meiotic genes (Figure $4 E$ ). Finally, to understand the actual nature of the block in spermatogenesis in NBMs, we examined histological sections of BM and NBM testes. Confirming previous observations [19, 26, 27], all stages of spermatogenesis could be observed in both BM and NBM animals (Supplementary Figure 15A, B), although it was not possible to obtain a precise quantitative information by this method. Interestingly, a very clear difference was observed in interstitial (Leydig) cell content, with much higher numbers in the BM compared to NBM testes (Supplementary Figure 15C,D).

In conclusion, our detailed analysis of NMR testes revealed that although both breeding and non-breeding males undergo spermatogenesis, non-breeders show signs of impaired post-meiotic sperm maturation at the transcriptomic level. This may lead to lower sperm numbers and impaired motility that could underlie their incapacity to breed. 


\section{Discussion}

415 In this study, we set out to define the molecular pathways that might underlie one of the principal features

416 of eusociality, namely the extreme socially-induced suppression of reproduction (reproductive skew) in

417 NMRs. We achieved this by comparison of brain and gonad transcriptomes in the different individuals

418 that make up the NMR social hierarchy, namely Qs (the only breeding females), NBFs, BMs and NBMs.

419 The comparative analysis of gene expression in the brain of different NMR social classes revealed that: 1)

420 the Q has a distinctive gene expression profile when compared to the rest of the colony; 2) both sexes of

421 non-breeding animals have nearly identical gene expression profiles in the brain; 3) several genes show

422 significant gene expression differences in comparisons between breeding animals (Qs vs BMs) and

423 between breeding animals versus non-breeding animals; 4) the Qs and NBFs have clear gene expression

424 differences in the brain, but this is not reflected in comparisons between BMs and NBMs; 5) the

425 dopaminergic pathway is identified as a major pathway that may be linked to the differences between

426 social classes of NMR colonies. More specifically, differences in TH expression (the rate limiting enzyme

427 for the synthesis of dopamine) were found in the hypothalamic areas of the brain which control prolactin

428 secretion [48]. Also, the catecholaminergic innervation (potentially dopaminergic) in the hippocampus

429 was strikingly high in breeders and showed a clear difference between breeders and non-breeders. In mice

430 or rats, there are sparse catecholaminergic afferents, which arise mainly from the ventral tegmental area

431 [79] and the locus coeruleus [80]. In NMR breeders, the origin of these afferents and their role in the

432 different behaviors between castes remains to be investigated. We believe that this potential role of

433 dopamine in NMR eusociality is a significant discovery that is consistent with findings in other eusocial

434 animals. Indeed, the dopaminergic pathway is a highly conserved system across vertebrates and some

435 invertebrates, with many important functions in the nervous system [81-86]. These include a crucial role

436 in the control of movement and reinforcement learning. Dopamine is also implicated in sexual arousal,

437 aggression, dominance, sleep, attention, working memory, hormonal regulation, and other functions [82,

438 87-90]. In eusocial insects and termites, the importance of dopamine in aggression, dominance and social 
(Diacamma sp. from Japan); and a positive correlation was observed between ovarian activity and the

442 level of dopamine in wasps (Polistes chinensis), bumble bees (Bombus terrestris), honeybees (Apis

443 mellifera L.), ants (Harpegnathos saltator) [91-96]. In the honeybee (Apis mellifera), tyrosine or royal

444 jelly-fed workers had higher brain dopamine levels than sucrose fed individuals, resulting in ovarian

445 development and the inhibition of foraging in the former [97]. Dietary and topical applications of

446 dopamine in queenless ant subordinate workers have also been shown to induce oocyte growth/activation

447 [93, 96]. In honey bee (Apis mellifera), queen pheromone have been shown to modulate dopamine

448 signaling pathways in the worker bees [98]. Furthermore, in male honeybees, juvenile hormone (JH),

449 which regulate development and reproduction, has been shown to increase the levels of dopamine in the

450 brain $[99,100]$. JH and brain dopamine levels have been shown to increase during sexual maturation, and

451 topical application of a $\mathrm{JH}$ analog increases the level of dopamine in the brain of male honeybees [101,

452 102]. These investigations indicate that the dopaminergic pathway can be crucially involved in the social

453 system, behavior, and reproductive suppression of many eusocial insects and termites. Our findings point

454 to the exciting possibility that dopamine-mediated mechanisms are also involved in maintaining

455 eusociality in the NMRs, suggesting that these pathways may represent a rather universal strategy for

456 eusociality across the Animal Kingdom.

457 The potential functional significance of the observed differences in dopamine pathways between the Q 458 and the non-breeders could be at several levels. One possible and well-established mechanism is the 459 action of dopamine on prolactin regulation (reviewed in $[48,103]$ ). In this pathway, dopamine (also 460 known as prolactin inhibiting factor), modulates the release of prolactin from the pituitary gland, and 461 prolactin itself acts on the hypothalamus to regulate the release of GnRH (Supplementary Figure 16B,C)

462 [104]. The plasma prolactin data, measured for the first time in this species, fits our model of elevated 463 PRL suppressing reproduction in non-breeders, because plasma PRL concentrations measured in spot 464 samples collected from most non-breeding NMRs often greatly exceeded values which would be 
considered clinical hyperprolactinemia in humans (Figure 1F). For example, levels of circulating PRL between 3 and $15 \mathrm{mg} / \mathrm{l}$ are considered necessary for maintaining normal reproductive function, and levels below and above are associated with an increased rate of infertility[105] - generally this equates to fasting concentrations above $25 \mathrm{ng} / \mathrm{ml}$ for women and $20 \mathrm{ng} / \mathrm{ml}$ for men [106]. Furthermore, plasma PRL in nonbreeders often also exceeded that of lactating queens (Figure $1 F$ ). We did fail to detect PRL in some 470 plasma samples from non-breeders. However, plasma PRL concentrations are known to exhibit circadian

471 variations, and any such pattern or synchrony amongst NMRs remains to be investigated in detail to avoid

472 sampling at times of low secretion. In mammals, hyperprolactinaemia is well known to be a major cause

473 of infertility, and plays a role in this context in lactational suppression of reproduction [107]. A higher

474 level of dopamine in the Q or BM is expected to decrease the release of prolactin. In turn, the absence or

475 decreased levels of prolactin may result in the normal pulsatile secretion of GnRH from the

476 hypothalamus, and subsequent release of LH and FSH from the pituitary gland. LH and FSH exert their

477 effects at the level of the gonad, stimulating sexual differentiation, follicular development, 478 spermatogenesis and release of the sex hormones estrogen (in females) and testosterone (in males)

479 (Supplementary Figure 16B). The low dopamine in non-breeding animals on the other hand, results in 480 elevated levels of prolactin, inhibiting the release of GnRH, and thus $\mathrm{LH}, \mathrm{FSH}$, estrogen, and testosterone, 481 and follicular development and spermatogenesis (Supplementary Figure 16C). The inhibitory effect of 482 dopamine on prolactin has been shown in many animals, and specific dopamine neurons (A12 and A14 483 cell groups, Supplementary Figure 16A) are known to regulate the release of prolactin. Interestingly, the 484 unique presence of cell bodies expressing RFRP-3 in the arcuate nucleus of NMRs, reported by [34] may 485 be of significance, as it could potentially allow interaction with the A12 dopaminergic cell groups that 486 regulate PRL section. Despite the differences in expression of genes involved in increasing dopamine in 487 the brain, we did not observe differences in the GnRH expression between breeding and non-breeding 488 NMR brains (both males and females). However, this would fit with predictions based on previous NMR 489 studies that showed similar numbers of immunoreactive GnRH-1 cell bodies among breeders, non490 breeders, males and females [33]. This implies that GnRH is produced by all status groups, but only 
actually released in breeders, due to a block to its secretion in non-breeders. The neuropeptide kisspeptin may play a role in this process among females. Kisspeptin is well known to influence the hypothalamopituitary-gonadal axis by direct actions on GnRH-1 neurons [33]. Zhou et al. found that breeding females NMRs had increased numbers of kisspeptin immunoreactive cell bodies in the anterior periventricular nucleus and rostral periventricular region of the third ventricle (RP3V). This suggests a role for kisspeptin in the hypogonadotrophic state in female NMRs, acting via mechanisms similar to those that underlie puberty and seasonal breeding in other species. Our observation of small increases in expression of Kiss-1 and its receptor (Kiss-1R) (the latter being statistically significant) in Qs versus NBFs supports this hypothesis. Furthermore, elevated prolactin is known to have a suppressing effect on kisspeptin (and hence GnRH), which would fit a model of suppression involving dopamine pathways. Brown et al. (2014) showed that administration of prolactin to mice caused Kiss-1 mRNA to be suppressed in the RP3V (the region with increased kisspeptin immunoreactive cell bodies in Q NMRs). It remains to be determined how the elevated levels of RFRP-3 (GnIH) in the brain of non-breeders, reported by [34], act in this mechanism that ultimately inhibits GnRH secretion. Interestingly, elevated prolactin has been implicated in many studies as a factor mediating both parental and alloparental care, affiliative and other sociosexual behaviours, in birds and mammals, including rodents and primates [108]._t is thus tempting to speculate that elevated prolactin in NMRs may play a central role in both cooperative behaviour and reproductive suppression.

The hypothalamic block to reproduction in NMRs ultimately results in the well-documented lack of

511 gonadal development in both male and female non-breeders [18, 19, 27]. Not surprisingly, given the large

512 anatomical differences, gene expression profiling of the ovary identified several DEGs between the Qs

513 and NBFs. One of the important differences in gene expression was the significantly higher expression of

514 aromatase in Qs ovary (top10 DEGs), the key enzyme in the production of estrogen. This difference in

515 aromatase expression indicates the ability of the Qs ovary to produce estrogen, and the low production of

516 ovarian estrogen in NBFs. Aromatase knockout mice display underdeveloped external genitalia and uteri, 
and precocious depletion of ovarian follicles, and anovulation, with development of the mammary glands approximately that of prepubertal WT female mice [109-111]. These features of aromatase knockout mice mimic most of the anatomical and physiological and endocrine differences that were observed between the Qs and NBFs of NMR [18, 19, 24]. The enrichment analysis on DEGs also highlights the difference between the Q and NBF ovaries. The small, pre-pubertal ovaries of NBFs mostly contain primordial and primary follicles that are arrested at the meiosis I prophase. In contrast, the fully functional ovary of the

Qs contains follicles at all stages of development, including some polyovular follicles that may contain up

to three oocytes [112], and larger numbers of stromal cells (Supplementary Figure 10). The ovarian

stroma is a diverse mix of cell types that includes theca-interstitial cells, immune cells, blood vessels, smooth muscle cells, and several types of extracellular matrix proteins [113-118]. The increased stromal cell content in Qs is reflected in the enrichment of GO terms (such as regulation of leukocyte differentiation, regulation of hematopoiesis, cell adhesion) for DEGs unregulated in the Qs. The NBF ovaries, with mostly primary follicles containing oocytes arrested at meiosis I, is reflected in GO terms (such as DNA methylation involved in gamete generation, sexual reproduction, meiotic nuclear division, meiotic cell cycle) for DEGs upregulated in the NBFs. Taken together, the expression analyses reported here is consistent with the observation that the Qs ovary is able to undergo oogenesis/folliculogenesis, ovulation and other sexual differentiation processes; whereas NBF ovaries are arrested at a pre-pubertal stage, lack the ability to produce estrogen, ovulate and undergo sexual differentiation (Supplementary Figure 16D). In the testis, our gene expression profiling of BM and NBMs revealed that there are no obvious

538 differences, unlike what was observed between Q vs NBF ovaries. This could be partly explained by the 539 fact that NBFs do not produce mature gametes but Q's do, whereas both BMs and NBMs NMRs undergo 540 spermatogenesis to produce gametes, and this is reflected in spermatogenesis gene expression profiles 541 seen in both. Nevertheless, some DEGs were identified that are known to play an important role in male 542 sexual differentiation, reproduction, and responses to steroids. In particular, the enrichment of GO term 
543 involved in male reproduction (meiotic cell cycle, male genitalia development, gamete generation,

544 spermatogenesis, and sperm motility) for upregulated DEGs in BMs includes genes such as Prm1, Prm2,

545 Odf3 and Akap4, which have been shown to have a important role in mouse spermatogenesis. A decrease

546 in expression of genes such as Prm1, Prm2, Odf3 and Akap4 and related genes in NBMs, might

547 contribute for the observed reduction in sperm number and motility [28]. This is further complemented by

548 the low expression of genes that are involved in meiotic and post-meiotic stages of spermatogenesis in

549 non-breeding animals. Based on these observations, we suggest that the main difference between BMs

550 and NBMs spermatogenesis is related to post-meiotic and sperm maturation stages (Supplementary

551 Figure 3E), where NBMs fail to express critical genes at appropriate level at these stages (post-meiotic

552 and sperm maturation stages), resulting in low sperm count and impaired motility. Cytological

553 examination of testicular sections of breeding and non-breeding animals (Supplementary Figure 15),

554 confirms previous observations of a higher interstitial cell content in breeding animals compared to non-

555 breeding animals. At the gene expression level, we also observed a significant decrease ( 2 fold,

556 Additional file 29) in the expression of the LH receptor gene (Lhcgr) in NBMs compared to BMs. This

557 fits with predictions based on the reduction in interstitial (Leydig) cells numbers, together with previously

558 reported observations of lower concentrations of urinary testosterone and plasma LH in NBMs [20].

\section{Conclusion}

Our study reveals gene expression differences among male and female NMR reproductive castes. Our findings in brain, ovaries and testes provide some of the first insights into the potential molecular mechanisms that are important in reproduction suppression of NMR. The gene expression differences in the NMR brains follow social status rather than sex, with the $\mathrm{Q}$ having a unique gene expression pattern compared to the rest of the colony. In highlighting the potential importance of dopamine pathways in the

567 brain, and a possible role for hyperprolactinemia in mediating suppression, our findings significantly 568 advance the understanding of the basis of mammalian eusociality and cooperative breeding systems. In 

the ovaries, Qs express genes that are required for estrogen production, and thereby undergoes

570 oogenesis/folliculogenesis, ovulation and other sexual differentiation processes. In contrast, NBFs are

571 arrested at a pre-pubertal stage, and do not have the ability to produce ovarian estrogen, explaining their

572 failure to ovulate and subsequent reproductive incompetency. In the testis, both BMs and NBMs undergo

573 spermatogenesis, however NBMs fail to express genes required for post-meiotic sperm maturation, giving

574 explanation to lower sperm numbers, impaired motility, and breeding incapacity of NBMs. While many

575 questions remain, the phenotypic plasticity exhibited by the NMR also offers scope for understanding the

576 dynamics of reproductive activation when non-breeders transition into breeding state (NBF to Qs or NBM

577 to BM), in particular the role of epigenetics and other regulatory factors affecting the genes associated in

578 this transformation. 


\section{Animals}

598 NMRs were maintained at Queen Mary University of London in compliance with institutional guidelines.

599 All animals were born in captivity, kept under constant ambient temperature of $28-30^{\circ} \mathrm{C}$, and housed in

600 artificial burrow systems composed of interconnected perspex tubing with separate chambers for nesting,

601 food and toilet, simulating natural burrow conditions. They were fed an ad libitum diet of a variety of

602 chopped root vegetables such as sweet potato and turnip. Animal tissues were collected post mortem

603 (Additional file $1 A$ ) following euthanasia in full accordance with Institutional and National animal care

604 and use guidelines.

\section{Blood sampling for hormone assay}

607 Blood samples were obtained from $X N B F$, Queen BM, NBM naked mole-rats from among 13 captive

608 colonies from QMUL and UP. All blood samples collected at the University of Pretoria were with local

609 ethics committee clearance. Blood samples were collected between 11 h00 and $15 \mathrm{~h} 00$ as follows: The

610 animals were hand held and venous blood samples collected from the hind foot. Approximately 300-

$611500 \mathrm{ul}$ of blood was collected into heparinised micro-haematocrit tubes (University of Pretoria samples) or

612 into a heparinised $1 \mathrm{ml}$ syringe from trunk blood following euthanasia (QMUL) prior to tissue collection.

613 The blood was centrifuged at $500 \mathrm{~g}$ for 15 minutes and the plasma separated from the red cells and stored

614 at $-80^{\circ} \mathrm{C}$ until hormone assay.

\section{RNA extraction and sequencing}

617 NMR brain (excluding the olfactory bulb, the cerebellum, the medulla and the pons), ovary, and testis

618 were snap frozen by immersion into liquid nitrogen and subsequently stored at $-80^{\circ} \mathrm{C}$ prior to RNA

619 extraction. Snap frozen tissues were mechanically powdered and mixed to maintain the heterogeneity of

620 the sample. Total RNA was extracted from tissues using Qiagen miRNeasy kit (Qiagen, USA) following 
the manufacturer's recommendations. The quality of the extracted RNA was controlled using the Agilent Bioanalyzer (Agilent) and Qubit Fluorometric Quantitation (Thermo Fisher Scientific Inc.). $1 \mu \mathrm{g}$ of high quality RNA (with RNA Integrity Number $($ RIN) $>7$ ) were used for RNA sequencing. Total RNA, after polyadenylated RNA purification, was prepared for sequencing using Illumina Truseq library preparation protocol. For each sample, around 100 (Additional file 1B) million raw paired-end sequence reads (101 base pair long) were generated using Illumina HiSeq 2000 sequencing instrument. Data sets are available from NCBI GEO under accession number (\#\#\#\#).

\section{RNA sequence (RNA-seq) analysis}

630 The quality of the generated RNA sequence was evaluated using FastQC (version 0.11.2) [119]. Sequence

631 adapters, low quality reads, and overrepresented sequences (e.g. mitochondrial sequences) in the reads

632 were removed using Trimmomatic (version 0.32) [120], and the quality of the reads was checked again

633 using FastQC. Sequence that passed the quality assessment were aligned to the NMR genome (hetGla2,

634 Broad institute, 2012) using tophat2 (version 2.0.6, default parameters) [121], with bowtie2 (version

635 2.1.0) [122]. For each sample genome guided de-novo transcriptome assembly was performed using

636 Cufflinks (version 2.2.1, default parameters) [123] and assembled transcript from all samples were

637 merged using cuffmerge (cufflinks) to generate a master reference transcripts. Merged transcripts (master

638 transcripts) were annotated to gene name using mouse transcripts. Mouse cDNA were obtained from

639 Ensembl [124], and a mouse cDNA blast database was generated using blast (version 2.2.25) [125, 126].

640 Assembled and merged NMR transcripts (master transcript) were searched against mouse cDNA blast

641 database, and transcripts with e val $<=10^{\wedge}-5$, with a length of $>200 \mathrm{bp}$ were retained. Transcript

642 abundance level was generated using master reference transcripts generated by cufflinks and HTSeq

643 (version 0.5.3p9) [127]. The transcript level quantified using HTSeq was used as an input for further

644 processing using $\mathrm{R}$ software environment for statistical computing and graphics (version 3.2.2). Data

645 normalization, removal of batch effect and other variant was performed using EDASeq $\mathrm{R}$ package

646 (version 2.2.0) [128] and RUVseq package (Remove Unwanted Variation from RNA-Seq package) [129]. 
647 In short, read counts were normalized using EDASeq R package, and “in-silico empirical” negative

648 controls genes, for RUVseq package (RUVg normalization) were obtained by taking least significantly

649 DEGs based on a first-pass differential expression analysis performed prior to RUVg normalization.

650 RUVseq package (RUVg normalization) was then performed using the "in-silico empirical" negative

651 controls genes. Differential expression was performed using edgeR R package (version 3.10.5) [130],

652 using the negative binomial GLM approach, edgeR normalization on raw counts, and by considering a

653 design matrix that includes both the covariates of interest and the factors of unwanted variation. DEGs

654 with false discovery rate (FDR $<=0.05$, Benjamini-Hochberg multiple testing correction), expression level

$655>1 \mathrm{CPM}$ (counts per million), and log fold change $>1$ were retained and used for further processing, gene

656 ontology and pathway analysis.

\section{Gene ontology and pathway analysis}

659 Gene ontology and pathway analysis were performed using Metascape (metascape.org) [131], Enrichr

660 [132], and Ingenuity Pathway Analysis (IPA®, QIAGEN Redwood City, www.qiagen.com/ingenuity). In

661 Metascape, for each given gene list of DEGs, pathway and process enrichment analysis was carried out

662 with the following ontology sources: GO Biological Processes, GO Molecular Functions and KEGG

663 Pathway. All genes in the genome were used as the enrichment background. Terms with p-value $<0.01$,

664 minimum count 3 , and enrichment factor $>1.5$ (enrichment factor is the ratio between observed count and

665 the count expected by chance) are collected and grouped into clusters based on their membership

666 similarities [131]. P-values are calculated based on accumulative hypergeometric distribution, q-values

667 are calculated using the Benjamini-Hochberg procedure to account for multiple testing [131]. Kappa

668 scores were used as the similarity metric when performing hierarchical clustering on the enriched terms

669 and then sub-trees with similarity $>0.3$ are considered a cluster. The most statistically significant term

670 within a cluster is chosen as the one representing the cluster [131]. To further capture the relationship

671 among terms, a subset of enriched terms were selected and rendered as a network plot, where terms with

672 similarity $>0.3$ are connected by edges, with the best p-values from each of the 20 clusters [131]. Similar 
673 independent enrichment analysis was performed using Enrichr [132], to validate the outcome of

674 Metascape. The list of DEGs were used as input to Enrichr and the enrichment of GO Biological

675 Processes, GO Molecular Functions was investigated. The final result was sorted and plotted by using a

676 combined score. The combined score is a combination of the p-value and z-score calculated by

677 multiplying the two scores as follows: $\mathrm{c}=\log (\mathrm{p}) * \mathrm{z}$, where $\mathrm{c}$ is the combined score, $\mathrm{p}$ is the $\mathrm{p}$-value

678 computed using Fisher's exact test, and $\mathrm{z}$ is the $\mathrm{z}$-score computed to assess the deviation from the

679 expected rank [132].

681 Tissue preparation and immunofluorescence

682 NMRs were deeply anesthetized with $80 \mathrm{mg}$ pentobarbital (400 $\mu \mathrm{L}$ Euthatal, IP) and transcardially

683 perfused with $40 \mathrm{~g} / \mathrm{L}$ formaldehyde in PBS for $10 \mathrm{~min}(10 \mathrm{~mL} / \mathrm{min})$. Brains were dissected and kept at

$6844^{\circ} \mathrm{C}$ for $12 \mathrm{~h}$ in a PBS solution containing $15 \%$ sucrose before being flash frozen in isopentane $(1 \mathrm{~min}$ at -

$\left.68530^{\circ} \mathrm{C}\right)$ and stored at $-80^{\circ} \mathrm{C}$. Serial coronal sections $(30 \mu \mathrm{m}$ thick) were made with a cryostat (Leica,

686 France) and mounted onto Superfrost Plus slides. For each animal, the first section containing some

687 frontal cortex was taken as the origin of the numbering. Sections were stored dry at $-80^{\circ} \mathrm{C}$ until being

688 processed for immunolabelling.

689 Sections were equilibrated to $-20^{\circ} \mathrm{C}$ before a short additional fixation with $30 \mathrm{~g} / \mathrm{L}$ formaldehyde in PBS

690 for $5 \mathrm{~min}$ at RT. Formaldehyde was then neutralized with TBS (50 mM Tris, $150 \mathrm{mM} \mathrm{NaCl}, \mathrm{pH} 7.4)$ for 5

$691 \mathrm{~min}$ at $4^{\circ} \mathrm{C}$, before two consecutive steps of permeabilization of 5 min each at $4^{\circ} \mathrm{C}$, first in PBS

692 containing $0.5 \%(\mathrm{vol} / \mathrm{vol})$ Triton X-100, then in PBS with $10 \%$ (vol/vol) methanol. The sections were

693 rinsed with 70\% ( $\mathrm{vol} / \mathrm{vol})$ ethanol (EtOH70) and kept in the same solution for $10 \mathrm{~min}$ at RT before being

694 treated with $0.1 \%(\mathrm{w} / \mathrm{vol})$ Sudan Black B in EtOH70, for autofluorescence removal. After two quick

695 rinses with EtOH70 and one PBS wash, 30 min preblocking at $4{ }^{\circ} \mathrm{C}$ was achieved with the IF buffer ie

696 PBS, 2\% (w/vol) BSA, 0.2\% (vol/vol) Tween 20, $50 \mathrm{mM}$ glycine. The anti-TH rabbit polyclonal antibody

697 (Abcam, ab112) diluted 1/800 in IF buffer was incubated overnight at $4{ }^{\circ} \mathrm{C}$. Then the sections were rinsed

6983 times 10 minutes with PBS before being incubated with an Alexa488-coupled goat anti-rabbit 

30 min DAPI staining step (100 nM in PBS), sections were finally rinsed and mounted in Vectashield

701 (Vector Laboratories, USA). Image acquisition was carried out at the Cell and Tissue Imaging Platform of the Genetics and Developmental Biology Department (UMR3215/U934) of Institut Curie. The sections of the different animals were processed at the same time with the same parameters. Full views of the sections consisted of mosaics of pictures made with the $5 \mathrm{X}$ objective of an upright epifluorescence microscope (Zeiss). Views of the hippocampus were made with the 10X objective. Additional z-stack pictures $(1 \mu \mathrm{m}$ steps $)$ of the regions of interest were taken for quantification, with the $10 \mathrm{X}$ objective of an upright spinning disk confocal microscope (Roper/Zeiss). For each picture, 5 consecutive confocal optical sections were summed, then the average intensity of the region of interest was measured, and finally the measures for both hemispheres were averaged (when applicable).

711 Histology of testes and ovaries

712 Gonadal tissue samples were fixed by immersion in 4\% paraformaldehyde in saline within 15 minutes of

713 collection for a minimum of seven days. After fixation tissue samples were dehydrated and cleared using

714 standard histological methodology, before embedding in paraffin wax. Sections of 5-8 $\mu \mathrm{m}$ were cut and

715 stained for light microscopy with haematoxylin-eosin. Photomicrographs of sections were captured with a

716 QIClick ${ }^{\mathrm{TM}}$ CCD Camera (01-QICLICK-R-F-CLR-12; QImaging) linked to a DMRA2 light microscope

717 (Leica), using Volocity ${ }^{\circledR}$ v.6.3.1 image analysis software (Perkin-Elmer) running on an iMac computer

718 (27-inch with OS X Yosemite, version 10.10).

\section{Prolactin assay and validation}

721 Plasma prolactin concentrations were determined using a commercial enzyme-linked immunosorbent

722 assay (Elabscience(C) Guinea pig prolactin ELISA kit, Catalogue No: E-EL-GP0358) according to the

723 instructions in the manufacturer's user manual. In brief, $100 \mathrm{ml}$ of reference standard and diluted samples

724 (1/2 to $1 / 50$ in sample diluent) were transferred into coated wells of a $96-$ well micro-ELISA plate, 
725 respectively, and incubated for $90 \mathrm{~min}$ at $37^{\circ} \mathrm{C}$. Subsequently, all supernatant was removed, and the plate

726 patted dry. Immediately, $100 \mathrm{ml}$ of biotinylated detection antibody was added, and incubated for $60 \mathrm{~min}$

727 at $37^{\circ} \mathrm{C}$. The plate was washed 3 times, patted dry, and $100 \mathrm{ml}$ of horse radish peroxide (HRP) conjugate

728 were added and incubated for $30 \mathrm{~min}$ at $37^{\circ} \mathrm{C}$. Subsequently, the plate was washed 5 times with wash-

729 buffer, and patted dry. $90 \mathrm{ml}$ of substrate reagent were added, and incubated for $15 \mathrm{~min}$ at $37^{\circ} \mathrm{C}$. To

730 terminate the enzymatic reaction, $50 \mathrm{ml}$ of stop solution were added. Optical density was determined at

$731450 \mathrm{~nm}$ and results calculated using a best-fit curve. The sensitivity of the assay was $0.1 \mathrm{ng} / \mathrm{ml}$, the

732 detection range $0.16-20 \mathrm{ng} / \mathrm{ml}$, and coefficient of variation for repeatability was $<10 \%$.

733

734

735

736

737

738

739

740

741

742

743

744

745

746

747

748

749

750 


\section{Acknowledgments}

752 The authors would like to acknowledge the Cell and Tissue Imaging Platform of the Genetics and 753 Developmental Biology Department (UMR3215/U934) of Institut Curie, member of France-Bioimaging 754 (supported by ANR-10-INSB-04), particularly Olivier Renaud for help with light microscopy. We thank 755 all members of the Edith Heard team for stimulating discussions, technical and conceptual advice, notably

756 Mikael Attia and Ronan Chaligné. We thank Deborah Bourc'his, Nicolas Servant, and Emmanuel Barillot

757 from Institut Curie for their insightful comments on the manuscript. We would like to acknowledge the

758 Biological Services Unit staff at QMUL (Richard Rountree, Arthur Gatward, Paul Sroka) for care and 759 maintenance of animals and Cécile Reyes (Institut Curie, Genomics facility) for tissue fragmentation and 760 RNA extraction, and Professor Jenny Jarvis (University of Cape Town). We thank the Institut Curie 761 High-throughput sequencing platform (NGS platform) for generating all the sequencing data.

\section{Funding}

764 This work was supported by the International Blaise Pascal Chair to EHB, financed by the Région Ile-de765 France and administered by the Fondation de l'Ecole normale supérieure. The Cell and Tissue Imaging 766 Platform of the Genetics and Developmental Biology Department was supported by ANR-10-INSB-04.

767 The Institut Curie High-throughput sequencing platform (NGS platform) was supported by ANR-10768 EQPX-03 and ANR10-INBS-09-08 from the Agence Nationale de le Recherche and by the Canceropôle 769 Ile-de-France). L.M-P was funded by an FRM fellowship (SPF20151234950). Funding to EH was from 770 Labex DEEP (ANR-11-LBX-0044) part of the IDEX Idex PSL (ANR-10-IDEX-0001-02 PSL) and 771 ABS4NGS (ANR-11-BINF-0001).

\section{Availability of data and materials}

774 The datasets generated and/or analysed during the current study are available in the NCBI GEO

775 repository (https://www.ncbi.nlm.nih.gov/geo/), under series number \#\#\#\#\# 


\section{Author contributions}

778 EM conceived the study, designed experimental strategies, performed all bioinformatic analyses,

779 interpreted results, performed experiments and wrote the manuscript. LMP performed and interpreted

780 brain validation experiments, and participated in writing the manuscript. DG performed RNA extraction.

781 NCB collected plasma samples and funded hormonal work, while SBG and AG carried out hormone

782 assays. EHB designed experimental strategies, interpreted results, funded the project. CGF conceived the

783 study, designed experimental strategies, interpreted results, performed experiments and wrote the

784 manuscript. EH conceived the study, designed experimental strategies, interpreted results, and wrote the

785 manuscript.

786

787 Competing interests

788 The authors declare no competing interests.

$790 \quad$ Ethical approval

$791 \quad$ Not applicable

792

793 Consent for publication

794 Not applicable 


\begin{tabular}{|c|c|c|}
\hline 804 & NMR & Naked mole-rats \\
\hline 805 & $\mathrm{LH}$ & Luteinizing hormone \\
\hline 806 & $\mathrm{GnRH}$ & Gonadotrophin releasing hormone \\
\hline 807 & RNA-seq & RNA-sequencing \\
\hline 808 & PCA & Principal component analysis \\
\hline 809 & $\mathrm{Q}$ & Queen \\
\hline 810 & Qs_Tr & Queen technical replicate \\
\hline 811 & NBF & Non-breeding females \\
\hline 812 & $\mathrm{NBF}_{-} \mathrm{Tr}$ & Non breeding female technical replicate \\
\hline 813 & NBM & Non-breeding males \\
\hline 814 & $\mathrm{BM}$ & Breeding males \\
\hline 815 & $\mathrm{CPM}$ & Counts per million \\
\hline 816 & DEG & Differentially expressed genes \\
\hline 817 & GO & Gene ontology \\
\hline 818 & Q genes & Queen genes \\
\hline 819 & $\mathrm{TH} / \mathrm{Th}$ & Tyrosine hydroxylase \\
\hline 820 & FSH & Follicle-stimulating hormone \\
\hline 821 & PGCs & Primordial germ cells \\
\hline 822 & PRL & Prolactin \\
\hline 823 & bp & Base pair \\
\hline 824 & IF & Immunofluorescene \\
\hline 825 & PValue & p-value \\
\hline 826 & $\log \mathrm{CPM}$ & Counts per million in log scale \\
\hline 827 & $\log \mathrm{FC}$ & Log fold change \\
\hline 828 & FDR & False discovery rate \\
\hline 829 & $\mathrm{BH}$ & Benjamini-Hochberg \\
\hline
\end{tabular}




\section{$\underline{\text { References }}$}

\begin{tabular}{|c|c|c|}
\hline 836 & 1. & Szathmary E, Smith JM: The major evolutionary transitions. Nature 1995, 374:227-232. \\
\hline 837 & 2. & Nowak MA, Tarnita CE, Wilson EO: The evolution of eusociality. Nature 2010, 466:1057-1062. \\
\hline 838 & 3. & Wilson EO: The insect societies. Cambridge, Mass.: Belknap Press of Harvard University Press; 1971. \\
\hline 839 & 4. & Michener CD: Comparative Social Behavior of Bees. Annual Review of Entomology 1969, 14:299-342 \\
\hline 840 & 5. & Crespi BJ, Yanega D: The definition of eusociality. Oxford Journals, Behavioral Ecology 1995, 6:109-115. \\
\hline $\begin{array}{l}841 \\
842\end{array}$ & 6. & $\begin{array}{l}\text { Duffy JE, Morrison CL, Rios R: Multiple origins of eusociality among sponge-dwelling shrimps } \\
\text { (Synalpheus). Evolution 2000, 54:503-516. }\end{array}$ \\
\hline $\begin{array}{l}843 \\
844\end{array}$ & 7. & $\begin{array}{l}\text { Duffy JE, Macdonald KS: Kin structure, ecology and the evolution of social organization in shrimp: a } \\
\text { comparative analysis. Proc Biol Sci 2010, 277:575-584. }\end{array}$ \\
\hline $\begin{array}{l}845 \\
846\end{array}$ & 8. & $\begin{array}{l}\text { Jarvis JU: Eusociality in a mammal: cooperative breeding in naked mole-rat colonies. Science 1981, } \\
\text { 212:571-573. }\end{array}$ \\
\hline $\begin{array}{l}847 \\
848\end{array}$ & 9. & $\begin{array}{l}\text { Friedman DA, Gordon DM: Ant Genetics: Reproductive Physiology, Worker Morphology, and Behavior. } \\
\text { Annu Rev Neurosci 2016, 39:41-56. }\end{array}$ \\
\hline $\begin{array}{l}849 \\
850\end{array}$ & 10. & $\begin{array}{l}\text { J FE, H K: On Biomass and Trophic Structure of the Central Amazonian Rain Forest Ecosystem Biotropica } \\
\text { 1973, 5:2-14. }\end{array}$ \\
\hline 851 & 11. & Hölldobler B, Wilson EO: The ants. Cambridge, Mass.: Belknap Press of Harvard University Press; 1990. \\
\hline $\begin{array}{l}852 \\
853\end{array}$ & 12. & $\begin{array}{l}\text { M OR, CG F: African mole-rats: eusociality, relatedness and ecological constraints. In Ecology of social } \\
\text { evolution. Edited by J H, J K. Berlin, Germany: Springer; 2008: 205-220 }\end{array}$ \\
\hline $\begin{array}{l}854 \\
855\end{array}$ & 13. & $\begin{array}{l}\text { Faulkes CG, Bennett NC, Bruford MW, O'Brien HP, Aguilar GH, Jarvis JU: Ecological constraints drive } \\
\text { social evolution in the African mole-rats. Proc Biol Sci 1997, 264:1619-1627. }\end{array}$ \\
\hline $\begin{array}{l}856 \\
857\end{array}$ & 14. & $\begin{array}{l}\text { M. JJU, C. BN: Eusociality has evolved independently in two genera of bathyergid mole-rats - but occurs } \\
\text { in no other subterranean mammal. Behavioral Ecology and Sociobiology 1993, 33:253-260. }\end{array}$ \\
\hline $\begin{array}{l}858 \\
859 \\
860\end{array}$ & 15. & $\begin{array}{l}\text { RA. B: The ecology of naked mole-rat colonies: burrowing, food, and limiting factors. In The Biology of } \\
\text { the Naked Mole-Rat. Edited by PW S, JUM J, RD A. New Jersey: Princeton University Press; 1991: 97- } \\
136\end{array}$ \\
\hline $\begin{array}{l}861 \\
862 \\
863\end{array}$ & 16. & $\begin{array}{l}\text { EA. L, PW. S: Social organization of naked mole-rat colonies: Evidence for divisions of labor. In The } \\
\text { Biology of the Naked Mole-Rat. Edited by PW S, JUM J, RD A. New Jersey: Princeton University Press; } \\
\text { 1991: 275-336 }\end{array}$ \\
\hline $\begin{array}{l}864 \\
865\end{array}$ & 17. & $\begin{array}{l}\text { Buffenstein R: The naked mole-rat: a new long-living model for human aging research. J Gerontol A Biol } \\
\text { Sci Med Sci 2005, 60:1369-1377. }\end{array}$ \\
\hline $\begin{array}{l}866 \\
867\end{array}$ & 18. & $\begin{array}{l}\text { Faulkes CG, Abbott DH, Jarvis JU: Social suppression of ovarian cyclicity in captive and wild colonies of } \\
\text { naked mole-rats, Heterocephalus glaber. } J \text { Reprod Fertil 1990, 88:559-568. }\end{array}$ \\
\hline $\begin{array}{l}868 \\
869\end{array}$ & 19. & $\begin{array}{l}\text { Faulkes CG, Abbott DH, Jarvis JU, Sherriff FE: LH responses of female naked mole-rats, Heterocephalus } \\
\text { glaber, to single and multiple doses of exogenous GnRH. J Reprod Fertil 1990, 89:317-323. }\end{array}$ \\
\hline $\begin{array}{l}870 \\
871\end{array}$ & 20. & $\begin{array}{l}\text { Faulkes CG, Abbott DH: Social control of reproduction in breeding and non-breeding male naked mole-rats } \\
\text { (Heterocephalus glaber). J Reprod Fertil 1991, 93:427-435. }\end{array}$ \\
\hline $\begin{array}{l}872 \\
873\end{array}$ & 21. & $\begin{array}{l}\text { Faulkes CG, Abbott DH: Evidence that primer pheromones do not cause social suppression of reproduction } \\
\text { in male and female naked mole-rats (Heterocephalus glaber). J Reprod Fertil 1993, 99:225-230. }\end{array}$ \\
\hline $\begin{array}{l}874 \\
875\end{array}$ & 22. & $\begin{array}{l}\text { Clarke FM, Faulkes CG: Dominance and queen succession in captive colonies of the eusocial naked mole- } \\
\text { rat, Heterocephalus glaber. Proc Biol Sci 1997, 264:993-1000. }\end{array}$ \\
\hline $\begin{array}{l}876 \\
877\end{array}$ & 23. & $\begin{array}{l}\text { Clarke FM, Faulkes CG: Hormonal and behavioural correlates of male dominance and reproductive status } \\
\text { in captive colonies of the naked mole-rat, Heterocephalus glaber. Proc Biol Sci 1998, 265:1391-1399. }\end{array}$ \\
\hline $\begin{array}{l}878 \\
879 \\
880\end{array}$ & 24. & $\begin{array}{l}\text { Margulis SW, Saltzman W, Abbott DH: Behavioral and hormonal changes in female naked mole-rats } \\
\text { (Heterocephalus glaber) following removal of the breeding female from a colony. Horm Behav 1995, } \\
\text { 29:227-247. }\end{array}$ \\
\hline $\begin{array}{l}881 \\
882\end{array}$ & 25. & $\begin{array}{l}\text { O'Riain MJ, Jarvis JU, Alexander R, Buffenstein R, Peeters C: Morphological castes in a vertebrate. Proc } \\
\text { Natl Acad Sci U S A 2000, 97:13194-13197. }\end{array}$ \\
\hline $\begin{array}{l}883 \\
884\end{array}$ & 26. & $\begin{array}{l}\text { Dengler-Crish CM, Catania KC: Phenotypic plasticity in female naked mole-rats after removal from } \\
\text { reproductive suppression. J Exp Biol 2007, 210:4351-4358. }\end{array}$ \\
\hline $\begin{array}{l}885 \\
886\end{array}$ & 27. & $\begin{array}{l}\text { Faulkes CG, Abbott DH, Jarvis JU: Social suppression of reproduction in male naked mole-rats, } \\
\text { Heterocephalus glaber. J Reprod Fertil 1991, 91:593-604. }\end{array}$ \\
\hline 887 & 28. & $\begin{array}{l}\text { Faulkes CG, Trowell SN, Jarvis JU, Bennett NC: Investigation of numbers and motility of spermatozoa in } \\
\text { reproductively active and socially suppressed males of two eusocial African mole-rats, the naked mole-rat }\end{array}$ \\
\hline
\end{tabular}


(Heterocephalus glaber) and the Damaraland mole-rat (Cryptomys damarensis). J Reprod Fertil 1994, 100:411-416.

29. Legan SJ, Karsch FJ: Neuroendocrine regulation of the estrous cycle and seasonal breeding in the ewe. Biol Reprod 1979, 20:74-85.

30. Glasier A, McNeilly AS, Baird DT: Induction of ovarian activity by pulsatile infusion of LHRH in women with lactational amenorrhoea. Clin Endocrinol (Oxf) 1986, 24:243-252.

31. Abbott DH, Hodges JK, George LM: Social status controls LH secretion and ovulation in female marmoset monkeys (Callithrix jacchus). J Endocrinol 1988, 117:329-339.

32. Goodman RL, Lehman MN: Kisspeptin neurons from mice to men: similarities and differences. Endocrinology 2012, 153:5105-5118.

33. Zhou S, Holmes MM, Forger NG, Goldman BD, Lovern MB, Caraty A, Kallo I, Faulkes CG, Coen CW: Socially regulated reproductive development: analysis of GnRH-1 and kisspeptin neuronal systems in cooperatively breeding naked mole-rats (Heterocephalus glaber). J Comp Neurol 2013, 521:3003-3029.

34. Peragine DE, Pokarowski M, Mendoza-Viveros L, Swift-Gallant A, Cheng HM, Bentley GE, Holmes MM: RFamide-related peptide-3 (RFRP-3) suppresses sexual maturation in a eusocial mammal. Proc Natl Acad Sci U S A 2017, 114:1207-1212.

35. Toth AL, Varala K, Henshaw MT, Rodriguez-Zas SL, Hudson ME, Robinson GE: Brain transcriptomic analysis in paper wasps identifies genes associated with behaviour across social insect lineages. Proc Biol Sci 2010, 277:2139-2148.

36. Ferreira PG, Patalano S, Chauhan R, Ffrench-Constant R, Gabaldon T, Guigo R, Sumner S: Transcriptome analyses of primitively eusocial wasps reveal novel insights into the evolution of sociality and the origin of alternative phenotypes. Genome Biol 2013, 14:R20.

37. Standage DS, Berens AJ, Glastad KM, Severin AJ, Brendel VP, Toth AL: Genome, transcriptome and methylome sequencing of a primitively eusocial wasp reveal a greatly reduced DNA methylation system in a social insect. Mol Ecol 2016, 25:1769-1784.

38. Simola DF, Wissler L, Donahue G, Waterhouse RM, Helmkampf M, Roux J, Nygaard S, Glastad KM, Hagen DE, Viljakainen L, et al: Social insect genomes exhibit dramatic evolution in gene composition and regulation while preserving regulatory features linked to sociality. Genome Res 2013, 23:1235-1247.

39. Kapheim KM, Pan H, Li C, Salzberg SL, Puiu D, Magoc T, Robertson HM, Hudson ME, Venkat A, Fischman BJ, et al: Social evolution. Genomic signatures of evolutionary transitions from solitary to group living. Science 2015, 348:1139-1143.

40. Sadd BM, Barribeau SM, Bloch G, de Graaf DC, Dearden P, Elsik CG, Gadau J, Grimmelikhuijzen CJ, Hasselmann M, Lozier JD, et al: The genomes of two key bumblebee species with primitive eusocial organization. Genome Biol 2015, 16:76.

41. Smith CR, Toth AL, Suarez AV, Robinson GE: Genetic and genomic analyses of the division of labour in insect societies. Nat Rev Genet 2008, 9:735-748.

42. Hunt GJ, Gadau JR: Editorial: Advances in Genomics and Epigenomics of Social Insects. Front Genet 2016, 7:199.

43. Yu C, Li Y, Holmes A, Szafranski K, Faulkes CG, Coen CW, Buffenstein R, Platzer M, de Magalhaes JP, Church GM: RNA sequencing reveals differential expression of mitochondrial and oxidation reduction genes in the long-lived naked mole-rat when compared to mice. PLoS One 2011, 6:e26729.

44. Davies KT, Bennett NC, Tsagkogeorga G, Rossiter SJ, Faulkes CG: Family Wide Molecular Adaptations to Underground Life in African Mole-Rats Revealed by Phylogenomic Analysis. Mol Biol Evol 2015, 32:3089-3107.

45. Fang X, Seim I, Huang Z, Gerashchenko MV, Xiong Z, Turanov AA, Zhu Y, Lobanov AV, Fan D, Yim $\mathrm{SH}$, et al: Adaptations to a subterranean environment and longevity revealed by the analysis of mole rat genomes. Cell Rep 2014, 8:1354-1364.

46. Holmes MM, Goldman BD, Goldman SL, Seney ML, Forger NG: Neuroendocrinology and sexual differentiation in eusocial mammals. Front Neuroendocrinol 2009, 30:519-533.

47. Bjorklund A, Dunnett SB: Dopamine neuron systems in the brain: an update. Trends Neurosci 2007, 30:194-202.

48. Ben-Jonathan N, Hnasko R: Dopamine as a prolactin (PRL) inhibitor. Endocr Rev 2001, 22:724-763.

49. Jung D, Kee K: Insights into female germ cell biology: from in vivo development to in vitro derivations. Asian J Androl 2015, 17:415-420.

50. Bukovsky A, Caudle MR, Svetlikova M, Wimalasena J, Ayala ME, Dominguez R: Oogenesis in adult mammals, including humans: a review. Endocrine 2005, 26:301-316. 
51. Reiss M, Sants H: Behaviour and Social Organisation. 1987.

946 52. Pan H, O'Brien M J, Wigglesworth K, Eppig JJ, Schultz RM: Transcript profiling during mouse oocyte development and the effect of gonadotropin priming and development in vitro. Dev Biol 2005, 286:493506.

53. Pan L, Gong W, Zhou Y, Li X, Yu J, Hu S: A comprehensive transcriptomic analysis of infant and adult mouse ovary. Genomics Proteomics Bioinformatics 2014, 12:239-248.

54. Simpson ER, Zhao Y, Agarwal VR, Michael MD, Bulun SE, Hinshelwood MM, Graham-Lorence S, Sun T, Fisher CR, Qin K, Mendelson CR: Aromatase expression in health and disease. Recent Prog Horm Res 1997, 52:185-213; discussion 213-184.

55. Simpson ER, Mahendroo MS, Means GD, Kilgore MW, Hinshelwood MM, Graham-Lorence S, Amarneh B, Ito Y, Fisher CR, Michael MD, et al.: Aromatase cytochrome P450, the enzyme responsible for estrogen biosynthesis. Endocr Rev 1994, 15:342-355.

56. Griswold MD: Spermatogenesis: The Commitment to Meiosis. Physiol Rev 2016, 96:1-17.

57. Griswold MD, Oatley JM: Concise review: Defining characteristics of mammalian spermatogenic stem cells. Stem Cells 2013, 31:8-11.

58. J W, JB S, CM L: Mammalian spermatogenesis. Func Dev Embryol 2007, 1:99-117.

59. Ramaswamy S, Weinbauer GF: Endocrine control of spermatogenesis: Role of FSH and LH/ testosterone. Spermatogenesis 2014, 4:e996025.

60. Themmen APN, Huhtaniemi IT: Mutations of gonadotropins and gonadotropin receptors: elucidating the physiology and pathophysiology of pituitary-gonadal function. Endocr Rev 2000, 21:551-583.

61. Gospodarowicz D: Properties of the luteinizing hormone receptor of isolated bovine corpus luteum plasma membranes. J Biol Chem 1973, 248:5042-5049.

62. Rivero-Muller A, Potorac I, Pintiaux A, Daly AF, Thiry A, Rydlewski C, Nisolle M, Parent AS, Huhtaniemi I, Beckers A: A novel inactivating mutation of the LH/chorionic gonadotrophin receptor with impaired membrane trafficking leading to Leydig cell hypoplasia type 1. Eur J Endocrinol 2015, 172:K2736.

63. Ascoli M, Fanelli F, Segaloff DL: The lutropin/choriogonadotropin receptor, a 2002 perspective. Endocr Rev 2002, 23:141-174.

64. Sassone-Corsi P: Unique chromatin remodeling and transcriptional regulation in spermatogenesis. Science 2002, 296:2176-2178.

65. Steger K: Transcriptional and translational regulation of gene expression in haploid spermatids. Anat Embryol (Berl) 1999, 199:471-487.

66. Balhorn R: The protamine family of sperm nuclear proteins. Genome Biol 2007, 8:227.

67. Steger K, Failing K, Klonisch T, Behre HM, Manning M, Weidner W, Hertle L, Bergmann M, Kliesch S: Round spermatids from infertile men exhibit decreased protamine-1 and -2 mRNA. Hum Reprod 2001, 16:709-716.

68. Bjorndahl L, Kvist U: Human sperm chromatin stabilization: a proposed model including zinc bridges. $\mathrm{Mol}$ Hum Reprod 2010, 16:23-29.

69. Carrell DT, Emery BR, Hammoud S: Altered protamine expression and diminished spermatogenesis: what is the link? Hum Reprod Update 2007, 13:313-327.

70. Luke L, Vicens A, Tourmente M, Roldan ER: Evolution of protamine genes and changes in sperm head phenotype in rodents. Biol Reprod 2014, 90:67.

71. Imken L, Rouba H, El Houate B, Louanjli N, Barakat A, Chafik A, McElreavey K: Mutations in the protamine locus: association with spermatogenic failure? Mol Hum Reprod 2009, 15:733-738.

72. Ravel C, Chantot-Bastaraud S, El Houate B, Berthaut I, Verstraete L, De Larouziere V, Lourenco D, Dumaine A, Antoine JM, Mandelbaum J, et al: Mutations in the protamine 1 gene associated with male infertility. Mol Hum Reprod 2007, 13:461-464.

73. Clark AG, Civetta A: Evolutionary biology. Protamine wars. Nature 2000, 403:261, 263.

74. Petersen C, Aumuller G, Bahrami M, Hoyer-Fender S: Molecular cloning of Odf3 encoding a novel coiledcoil protein of sperm tail outer dense fibers. Mol Reprod Dev 2002, 61:102-112.

75. Baltz JM, Williams PO, Cone RA: Dense fibers protect mammalian sperm against damage. Biol Reprod 1990, 43:485-491.

76. Miki K, Willis WD, Brown PR, Goulding EH, Fulcher KD, Eddy EM: Targeted disruption of the Akap4 gene causes defects in sperm flagellum and motility. Dev Biol 2002, 248:331-342. proteins in sperm with reduced motility. Asian J Androl 2007, 9:641-649. 
78. Lardenois A, Gattiker A, Collin O, Chalmel F, Primig M: GermOnline 4.0 is a genomics gateway for germline development, meiosis and the mitotic cell cycle. Database (Oxford) 2010, 2010:baq030.

79. Gasbarri A, Sulli A, Packard MG: The dopaminergic mesencephalic projections to the hippocampal formation in the rat. Prog Neuropsychopharmacol Biol Psychiatry 1997, 21:1-22.

80. Kempadoo KA, Mosharov EV, Choi SJ, Sulzer D, Kandel ER: Dopamine release from the locus coeruleus to the dorsal hippocampus promotes spatial learning and memory. Proc Natl Acad Sci U S A 2016, 113:14835-14840.

81. Waddell S: Dopamine reveals neural circuit mechanisms of fly memory. Trends Neurosci 2010, 33:457464.

82. Wicker-Thomas C, Hamann M: Interaction of dopamine, female pheromones, locomotion and sex behavior in Drosophila melanogaster. J Insect Physiol 2008, 54:1423-1431.

83. Cottrell GA: Occurrence of dopamine and noradrenaline in the nervous tissue of some invertebrate species. Br J Pharmacol Chemother 1967, 29:63-69.

84. Kindt KS, Quast KB, Giles AC, De S, Hendrey D, Nicastro I, Rankin CH, Schafer WR: Dopamine mediates context-dependent modulation of sensory plasticity in C. elegans. Neuron 2007, 55:662-676.

85. Carlsson A: Thirty years of dopamine research. Adv Neurol 1993, 60:1-10.

86. Schultz W: Responses of midbrain dopamine neurons to behavioral trigger stimuli in the monkey. $J$ Neurophysiol 1986, 56:1439-1461.

87. Joshua M, Adler A, Bergman H: The dynamics of dopamine in control of motor behavior. Curr Opin Neurobiol 2009, 19:615-620.

88. Dayan P, Balleine BW: Reward, motivation, and reinforcement learning. Neuron 2002, 36:285-298

89. Wise RA: Dopamine, learning and motivation. Nat Rev Neurosci 2004, 5:483-494.

90. Heinz A, Schlagenhauf F: Dopaminergic dysfunction in schizophrenia: salience attribution revisited. Schizophr Bull 2010, 36:472-485.

91. Sasaki K, Yamasaki K, Nagao T: Neuro-endocrine correlates of ovarian development and egg-laying behaviors in the primitively eusocial wasp (Polistes chinensis). J Insect Physiol 2007, 53:940-949.

92. Penick CA, Brent CS, Dolezal K, Liebig J: Neurohormonal changes associated with ritualized combat and the formation of a reproductive hierarchy in the ant Harpegnathos saltator. J Exp Biol 2014, 217:14961503.

93. Okada Y, Sasaki K, Miyazaki S, Shimoji H, Tsuji K, Miura T: Social dominance and reproductive differentiation mediated by dopaminergic signaling in a queenless ant. J Exp Biol 2015, 218:1091-1098.

94. Bloch G, Simon T, Robinson GE, Hefetz A: Brain biogenic amines and reproductive dominance in bumble bees (Bombus terrestris). J Comp Physiol A 2000, 186:261-268.

95. JW. H, Woodring J: Elevated brain dopamine levels associated with ovary development in queenless worker honeybees (Apis mellifera L) Comparative Biochemistry and Physiology 1995, 111C:271-279.

96. T. D, Simoes ZLP, Bitondi MMG: Dietary dopamine causes ovary activation in queenless Apis mellifera workers. Apidologie, Springer Verlag 2003, 34:281-289.

97. Matsuyama S, Nagao T, Sasaki K: Consumption of tyrosine in royal jelly increases brain levels of dopamine and tyramine and promotes transition from normal to reproductive workers in queenless honey bee colonies. Gen Comp Endocrinol 2015, 211:1-8.

98. Beggs KT, Glendining KA, Marechal NM, Vergoz V, Nakamura I, Slessor KN, Mercer AR: Queen pheromone modulates brain dopamine function in worker honey bees. Proc Natl Acad Sci U S A 2007, 104:2460-2464.

99. Akasaka S, Sasaki K, Harano K, Nagao T: Dopamine enhances locomotor activity for mating in male honeybees (Apis mellifera L.). J Insect Physiol 2010, 56:1160-1166.

100. Mezawa R, Akasaka S, Nagao T, Sasaki K: Neuroendocrine mechanisms underlying regulation of mating flight behaviors in male honey bees (Apis mellifera L.). Gen Comp Endocrinol 2013, 186:108-115.

101. S. DOT, A. R, W. E: Reactivation of juvenile hormone synthesis in adult drones of the honey bee,Apis mellifera carnica. Experientia, Springer 1995, 51:945-952.

102. Giray T, Robinson GE: Common endocrine and genetic mechanisms of behavioral development in male and worker honey bees and the evolution of division of labor. Proc Natl Acad Sci U S A 1996, 93:1171811722.

103. Fitzgerald P, Dinan TG: Prolactin and dopamine: what is the connection? A review article. $J$ Psychopharmacol 2008, 22:12-19.

104. Brown RS, Herbison AE, Grattan DR: Effects of Prolactin and Lactation on A15 Dopamine Neurones in the Rostral Preoptic Area of Female Mice. J Neuroendocrinol 2015, 27:708-717. 
105. Kauppila A, Martikainen H, Puistola U, Reinila M, L R: Hypoprolactinemia and ovarian function. Fertility and Sterility 1988

, 49:437-441.

106. Majumdar A, Mangal NS: Hyperprolactinemia. J Hum Reprod Sci 2013, 6:168-175.

107. Brown RS, Herbison AE, Grattan DR: Prolactin regulation of kisspeptin neurones in the mouse brain and its role in the lactation-induced suppression of kisspeptin expression. J Neuroendocrinol 2014, 26:898-908.

108. Snowdon CT, Ziegler TE: Variation in prolactin is related to variation in sexual behavior and contact affiliation. PLoS One 2015, 10:e120650.

109. Fisher CR, Graves KH, Parlow AF, Simpson ER: Characterization of mice deficient in aromatase (ArKO) because of targeted disruption of the cyp19 gene. Proc Natl Acad Sci U S A 1998, 95:6965-6970.

110. Toda K, Takeda K, Okada T, Akira S, Saibara T, Kaname T, Yamamura K, Onishi S, Shizuta Y: Targeted disruption of the aromatase P450 gene (Cyp19) in mice and their ovarian and uterine responses to 17betaoestradiol. J Endocrinol 2001, 170:99-111.

111. Hewitt SC, Winuthayanon W, Korach KS: What's new in estrogen receptor action in the female reproductive tract. J Mol Endocrinol 2016, 56:R55-71.

112. R. L: What is an hystricomorph? In The Biology of hystricomorph rodents. Edited by IW R, Weir BJ e. London: Zoological Society of London; 1974: 7-20

113. Kent J, Ryle M: Histochemical studies on three gonadotrophin-responsive enzymes in the infantile mouse ovary. J Reprod Fertil 1975, 42:519-536.

114. Paranko J, Pelliniemi LJ: Differentiation of smooth muscle cells in the fetal rat testis and ovary: localization of alkaline phosphatase, smooth muscle myosin, F-actin, and desmin. Cell Tissue Res 1992, 268:521-530.

115. Brannstrom M, Mayrhofer G, Robertson SA: Localization of leukocyte subsets in the rat ovary during the periovulatory period. Biol Reprod 1993, 48:277-286.

116. Best CL, Pudney J, Welch WR, Burger N, Hill JA: Localization and characterization of white blood cell populations within the human ovary throughout the menstrual cycle and menopause. Hum Reprod 1996, 11:790-797.

117. Magoffin DA: The ovarian androgen-producing cells: a 2001 perspective. Rev Endocr Metab Disord 2002, 3:47-53.

118. Berkholtz CB, Lai BE, Woodruff TK, Shea LD: Distribution of extracellular matrix proteins type I collagen, type IV collagen, fibronectin, and laminin in mouse folliculogenesis. Histochem Cell Biol 2006, 126:583-592.

119. S. A: FastQC: a quality control tool for high throughput sequence data. . Babraham Bioinformatics 2010.

120. Bolger AM, Lohse M, Usadel B: Trimmomatic: a flexible trimmer for Illumina sequence data. Bioinformatics 2014, 30:2114-2120.

121. Kim D, Pertea G, Trapnell C, Pimentel H, Kelley R, Salzberg SL: TopHat2: accurate alignment of transcriptomes in the presence of insertions, deletions and gene fusions. Genome Biol 2013, 14:R36.

122. Langmead B, Salzberg SL: Fast gapped-read alignment with Bowtie 2. Nat Methods 2012, 9:357-359.

123. Trapnell C, Williams BA, Pertea G, Mortazavi A, Kwan G, van Baren MJ, Salzberg SL, Wold BJ, Pachter L: Transcript assembly and quantification by RNA-Seq reveals unannotated transcripts and isoform switching during cell differentiation. Nat Biotechnol 2010, 28:511-515.

124. Yates A, Akanni W, Amode MR, Barrell D, Billis K, Carvalho-Silva D, Cummins C, Clapham P, Fitzgerald S, Gil L, et al: Ensembl 2016. Nucleic Acids Res 2016, 44:D710-716.

125. Camacho C, Coulouris G, Avagyan V, Ma N, Papadopoulos J, Bealer K, Madden TL: BLAST+: architecture and applications. BMC Bioinformatics 2009, 10:421.

126. Altschul SF, Gish W, Miller W, Myers EW, Lipman DJ: Basic local alignment search tool. J Mol Biol 1990, 215:403-410.

127. Anders S, Pyl PT, Huber W: HTSeq--a Python framework to work with high-throughput sequencing data. Bioinformatics 2015, 31:166-169.

128. Risso D, Schwartz K, Sherlock G, Dudoit S: GC-content normalization for RNA-Seq data. BMC Bioinformatics 2011, 12:480.

129. Risso D, Ngai J, Speed TP, Dudoit S: Normalization of RNA-seq data using factor analysis of control genes or samples. Nat Biotechnol 2014, 32:896-902.

130. Robinson MD, McCarthy DJ, Smyth GK: edgeR: a Bioconductor package for differential expression analysis of digital gene expression data. Bioinformatics 2010, 26:139-140. 
1112

1113

1114

1115

1116

1117

1118
131. Tripathi S, Pohl MO, Zhou Y, Rodriguez-Frandsen A, Wang G, Stein DA, Moulton HM, DeJesus P, Che J, Mulder LC, et al: Meta- and Orthogonal Integration of Influenza "OMICs" Data Defines a Role for UBR4 in Virus Budding. Cell Host Microbe 2015, 18:723-735.

132. Kuleshov MV, Jones MR, Rouillard AD, Fernandez NF, Duan Q, Wang Z, Koplev S, Jenkins SL, Jagodnik KM, Lachmann A, et al: Enrichr: a comprehensive gene set enrichment analysis web server 2016 update. Nucleic Acids Res 2016, 44:W90-97. 


\section{Figure 1}

A Gene expression differences in the brain

B Total number of differentially expressed genes in the brain

biokxiv preprint doi: https://doi.org/10.1101/209932; this version posted October 27, 2017. The copyright holder for this preprint (which was not breedinged by peer revieQuy)eisthe author/funder, who has granted bioRxiv a license to display the preprint ifoperpetuity. It is made available under
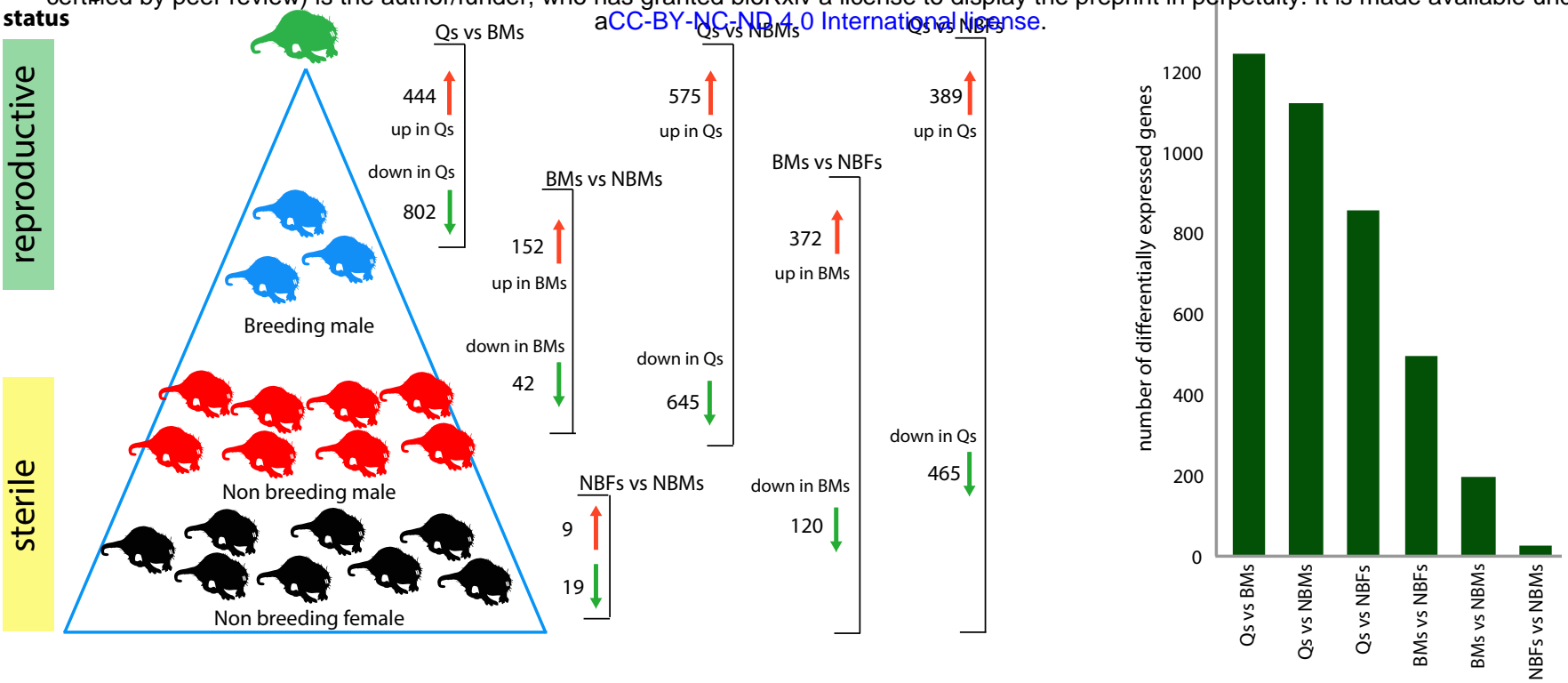

C Venn diagram of DEGs between brains of Qs vs NBFs and Qs vs NBMs

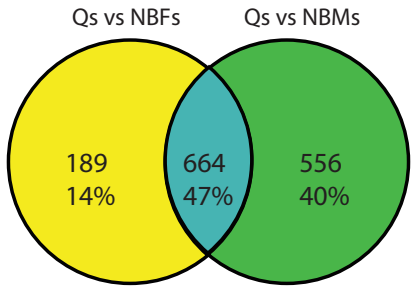

All differentially expressed genes
D Heatmap of enriched terms for queen genes (brain)

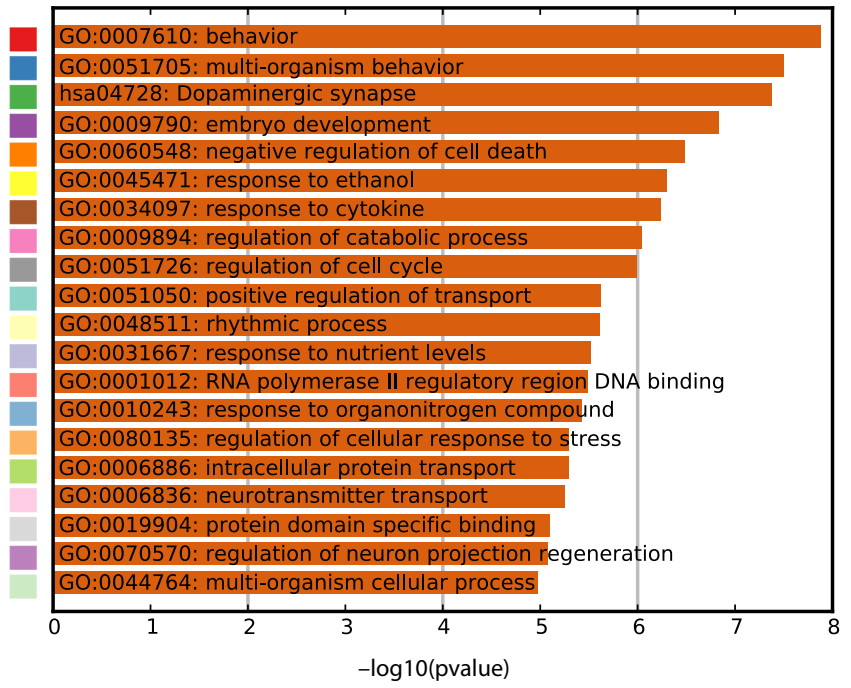

F Plasma prolactin level in breeding and non-breeding NMRs

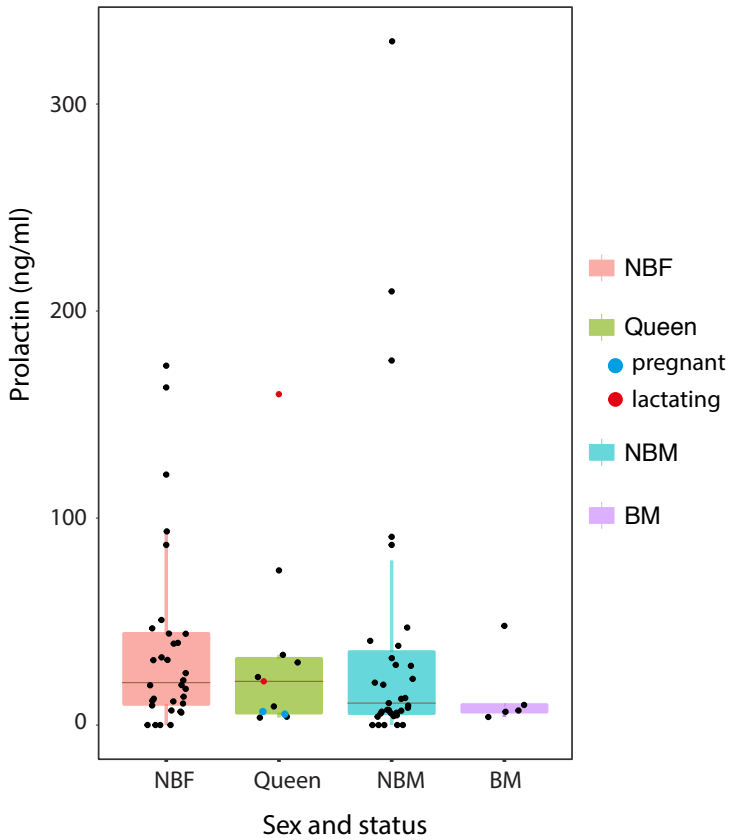



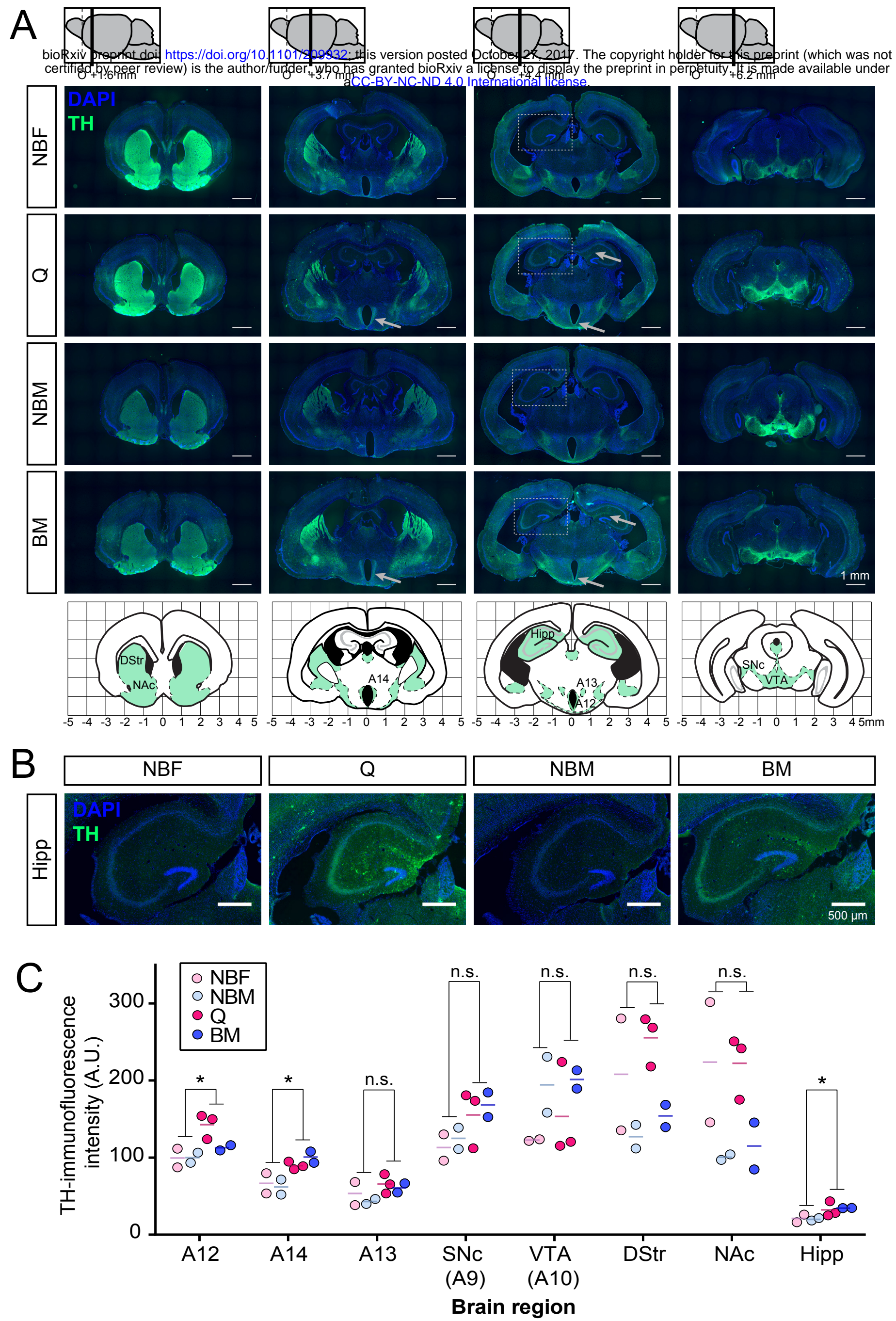


\section{Figure 3}

A PCA plot showing the clustering of $Q$ and NBF ovaries

B Hierarchical clustering of $\mathrm{Q}$ and NBF ovaries
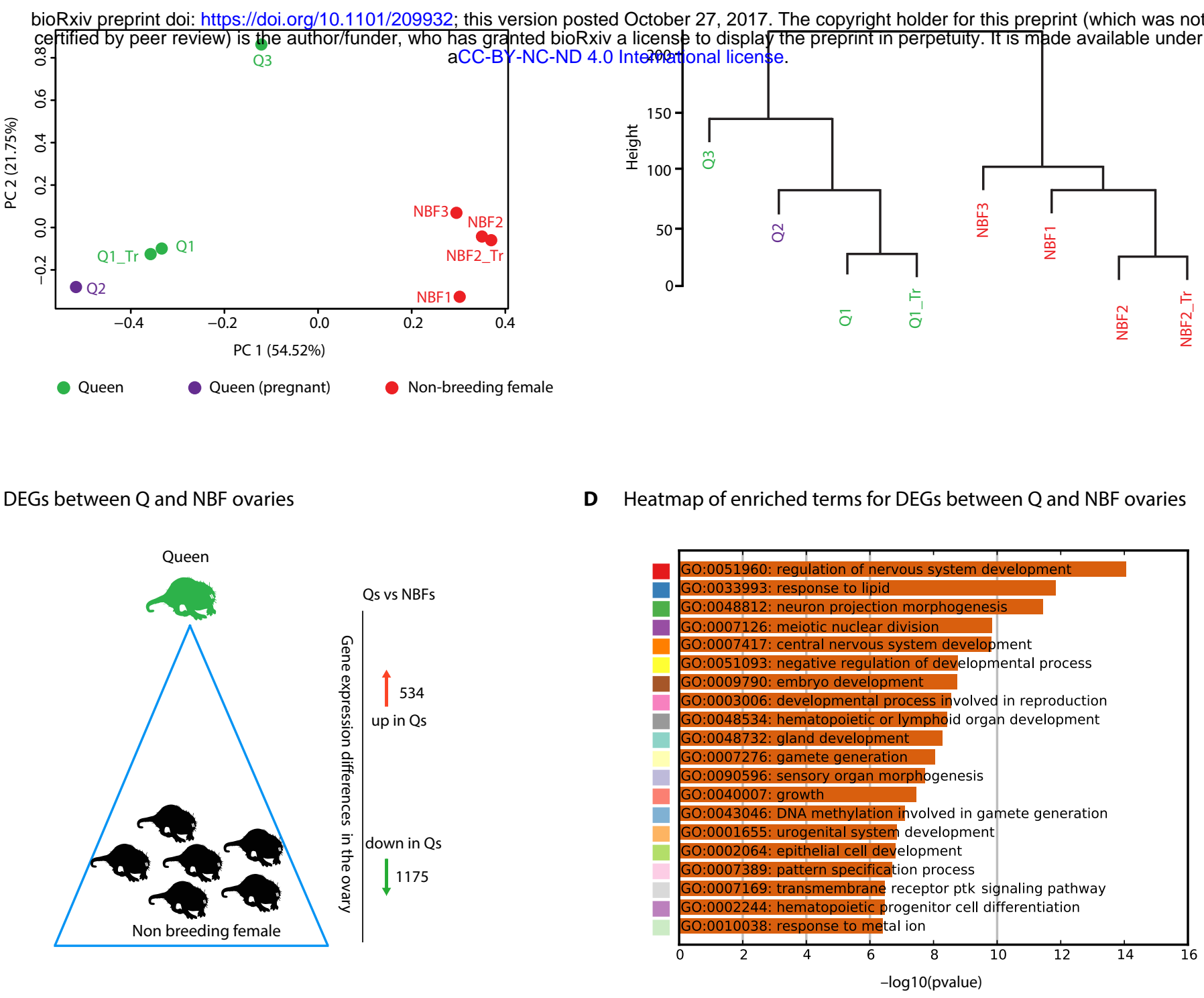


\section{Figure 4}

A PCA plot showing the clustering of BMs and NBMs

B Hierarchical clustering of BMs and NBMs testis gene expression profile

testis gene expression profile

bioRxiv preprint doi: https://doi.org/10.1101/209932; this version posted October 27, 2017. The copyright holder for this preprint (which was not certified by peer review) is the author/funder, who has granted bioRxiv a license to display the preprint in perpetuity. It is made available under

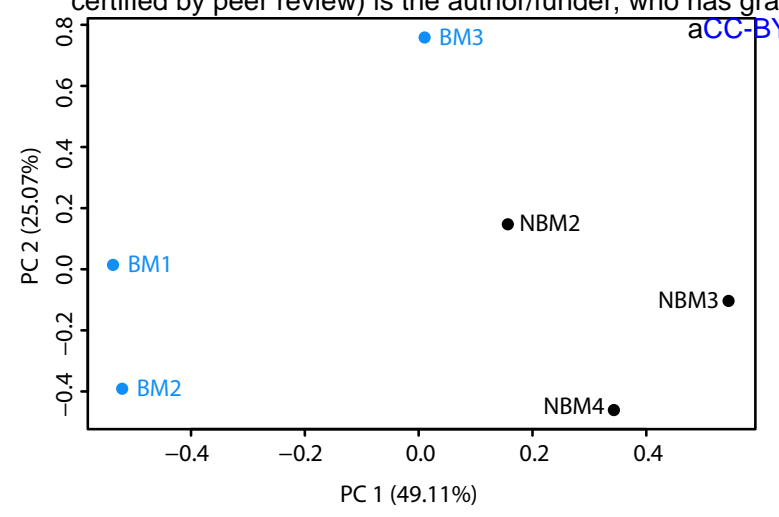

C DEGs between BMs and NBMs testis

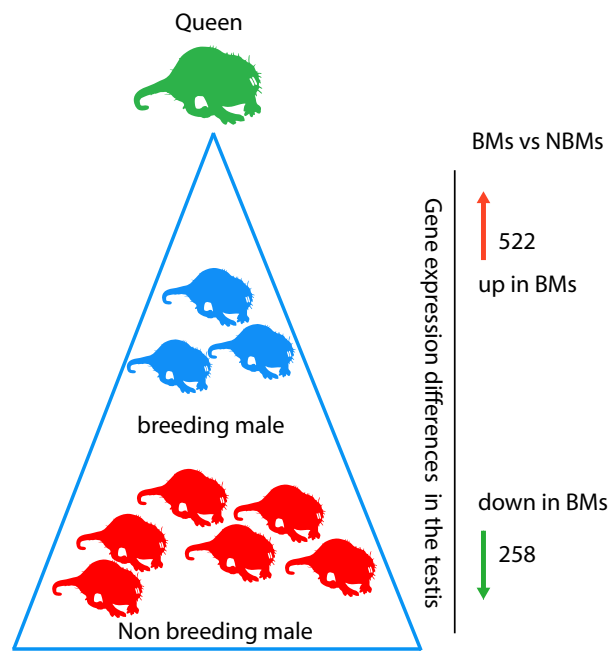

E Violin and box plots showing expression level of NMR genes that belong to different clusters of mouse spermatogenesis

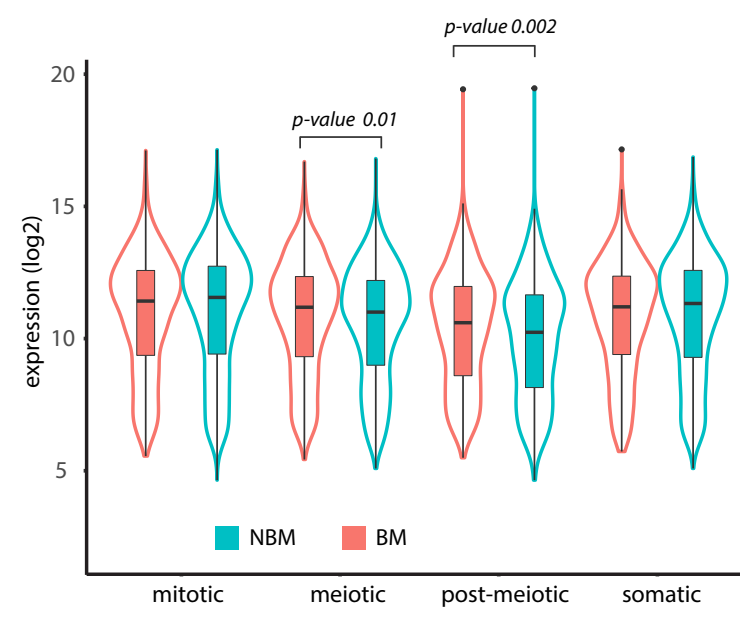

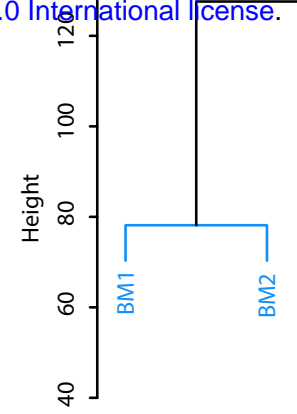

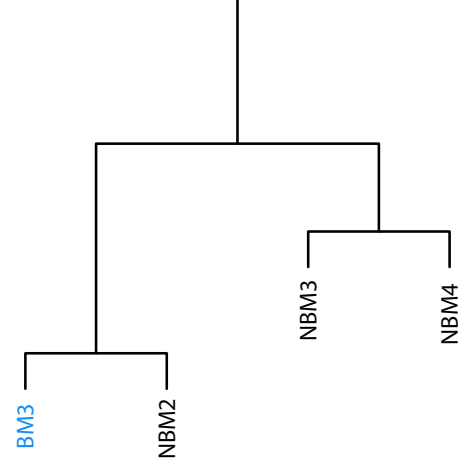

D Number of DEGs that belong to different stages of spermatogenesis

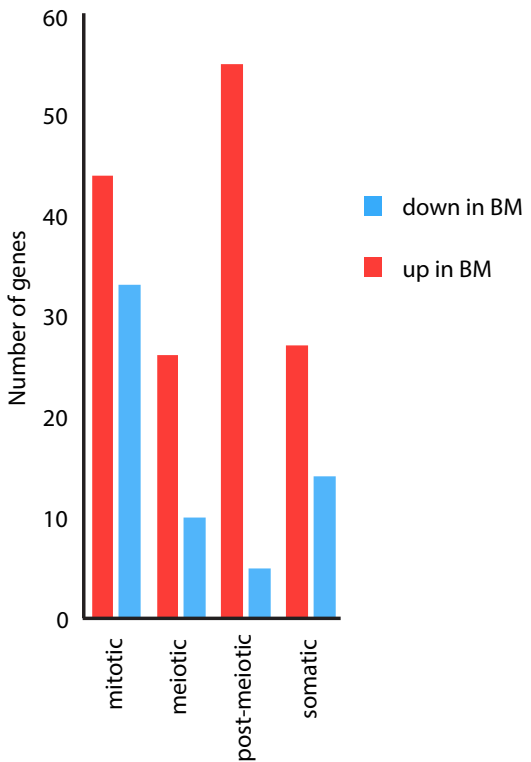

F Heatmap of enriched terms for DEGs between BMs and NBMs testis

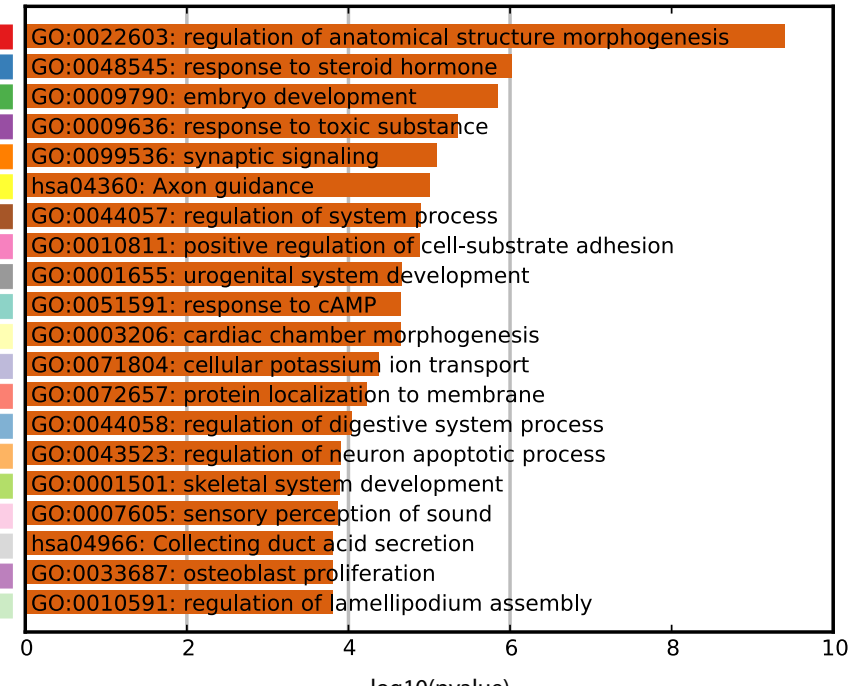

-log10(pvalue) 


\section{Figure legends}

3 Figure 1: Gene expression profile of naked mole rat colony members. A) Number of DEGs between

4 the different members of a naked mole rat colony brains. DEGs (FDR $<0.05$, log fold change $>1$ ) were

5 divided into up regulated (higher expression in one group) and down regulated (lower expression in one

6 group) and the final count is plotted. B) Total number of DEGs in each comparison (similar to Figure 1A,

7 but total number in each comparison plotted). C) Venn diagram showing DEGs that are common in the

8 comparison between Qs vs NBFs and Qs vs NBMs (Q genes, 661 genes in total), and genes that show

9 specific differential expression between Qs vs NBFs (192 genes) and Qs vs NBMs (560 genes). D)

10 Heatmap of enriched terms (Canonical Pathways, GO Biological Processes, Hallmark Gene Sets, KEGG

11 Pathway) for genes that show differential expression in the comparison between Qs vs NBFs and Qs vs

12 NBMs (Q genes). Significance of enrichment is indicated on the $\mathrm{x}$-axis in $-\log _{10}$ (p-value). The color code

13 on the $y$-axis is used to show the clustering and relation of the significantly enriched networks/terms

14 (shown in Figure 1E). Detailed list of enriched terms and families can be found in Additional file 3. E)

15 Network of enriched terms (Figure 1D) colored by cluster ID, indicating the relationship between the

16 different enriched terms (cluster ID color correspond to the color code shown in Figure 1D y-axis). Nodes

17 that share the same cluster are close to each other. Each circle node represents a term and the size of the

18 circle is proportional to the number of genes that fall into that term, and the identity of the cluster is

19 indicated by its color (nodes of the same color belong to the same cluster). Similar terms are linked by an

20 edge (the thickness of the edge represents the similarity score). One term from each cluster is selected to

21 have its term description shown as label. F) Plasma prolactin concentrations in breeding and non-breeding

22 NMRs in samples taken across thirteen colonies. Abbreviations: Q, queen; Q1_Tr, technical replicate for

23 Q1; NBF, non-breeding female; NBM, non-breeding male; BM, breeding male; DEGs, differentially

24 expressed genes. 
Figure 2:Tyrosine hydroxylase (TH) immunostaining of NMR brain sections. A) Representative

27 immunostaining of whole coronal sections for the four reproductive castes (NBF, non-breeding female;

28 NBM, non-breeding male; Q, Queen; BM, breeding male). The top panel indicates the coordinates of the 29 coronal sections relative to $\mathrm{O}$, the origin of slice numbering. Grey arrows point to the regions that show 30 differential staining (quantified in C). Dashed boxes indicate the hippocampal region. The bottom panel 31 represents the regions where TH-staining has been observed. (Labels refer to regions quantified in C: 32 DStr, dorsal striatum; Hipp, hippocampus; NAc, nucleus accumbens; SNc, substantia nigra compacta;

33 VTA, ventral tegmental area). B) TH immunofluorescence in the hippocampus. C) Intensity of the TH 34 staining in the different brain regions. Non-parametric Mann-Whitney test, ${ }^{*} \mathrm{p}<0.05$; n.s., non35 significant.

Figure 3) Gene expression profiles of $Q$ and NBF ovaries. A) Principal component analysis (PCA) plot showing the clustering of $\mathrm{Q}$ and NBF ovaries based on global expression profile. B) Hierarchical clustering of ovary samples using euclidean distance matrix computation and ward.D2 agglomeration method. C) number of DEGs between Q and NBF ovaries. D) Heatmap of enriched terms (Canonical

41 Pathways, GO Biological Processes, Hallmark Gene Sets, KEGG Pathway) for all DEGs colored by p42 values. Significance of enrichment is indicated on the $x$-axis in $-\log _{10}(p$-value). The color code on the y43 axis is used to show the clustering and relation of this networks (shown in Supplementary Figure 7C).

44 More information about the enriched terms can be found in Additional file 26A. (Q1_Tr and NBF2_Tr 45 indicate technical replicates for Q1 and NBF2 respectively)

47 Figure 4: Gene expression profile of breeding and non-breeding males testes. A) Principal 48 component analysis (PCA) plot showing the clustering of BMs and NBMs testis based on global gene 49 expression profile. B) Hierarchical clustering of testis samples using euclidean distance matrix 50 computation and ward.D2 agglomeration method. C) Number of DEGs between breeding and non51 breeding animals. D) Bar plot showing the number of NMR DEGs (BMs vs NBMs testes) that belong to 
52 different stages of mouse spermatogenesis. Gene clusters that are expressed at different stages of mouse

53 spermatogenesis were obtained from Germonline, and mapped to the DEGs that were identified in the

54 comparison between breeding and non-breeding NMR testes. The DEGs which map to different stages of

55 spermatogenesis were divided into up or down regulated, (if they show higher or lower expression in BM

56 respectively) and plotted. E) Violin and box plots showing the average expression level of all BMs and

57 NBMs genes (NMR testes) that were mapped to genes which belong to the different clusters (4 clusters)

58 of mouse spermatogenesis. Gene clusters that are expressed at different stages of mouse spermatogenesis

59 were obtained from Germonline, mapped to NMR BMs and NBMs testis expression data, and plotted (x-

60 axis, cluster name; y-axis: average expression level in $\log _{2}$ scale). F) Heatmap of enriched terms

61 (Canonical Pathways, GO Biological Processes, Hallmark Gene Sets, KEGG Pathway) for all DEGs

62 colored by p-values (generated using Metascape). Significance of enrichment is indicated on the $\mathrm{x}$-axis in

$63-\log _{10}(\mathrm{p}$-value $)$. The color code on the y-axis is used to show the clustering and relation of this network

64 (shown in Supplementary Figure 12C). Detailed list of enriched terms and genes that belong to these

65 terms is provided in Additional file $30 \mathrm{~A}$.

66

67

68

69

70

71

72

73

74

75

76

77 


\section{Supplementary Figure legends}

Supplementary Figure 1: A) Principal component analysis (PCA) plots showing the clustering of different NMR colony members based on global brain gene expression profile. B) Cluster dendrogram showing hierarchical clustering of the NMR brain gene expression. Hierarchical clustering was generated using euclidean distance matrix computation and ward.D2 agglomeration method. C) Enriched biological process terms for DEGs that are common in the comparison between Qs vs NBMs and Qs vs NBMs (Q genes) generated using Enrichr. The length of the bar represents the significance of that specific gene-set or term, and the color intensity provides additional information about the significance (the brighter the color, the more significant that term is). Statistical information used to generate the graph including the pvalue and other enriched terms are provided in Additional file 18. D) Enriched molecular functions terms for DEGs that are common in the comparison between Qs vs NBMs and Qs vs NBMs (Q genes) generated using Enrichr. The length of the bar represents the significance of that specific gene-set or term, and the color intensity provides additional information about the significance (the brighter the color, the other enriched terms are provided in Additional file 19.

\section{Supplementary Figure 2: Gene expression differences between the brains of Qs and NBFs. A)}

96 Principal component analysis (PCA) plot showing the clustering of Qs and NBFs brains based global

97 gene expression profile. B) Cluster heatmap of Qs and NBFs brain gene expression. Sample distance was

98 calculated euclidean distance matrix computation and cluster agglomeration was done using ward.D2

99 method. Heatmap color indicate the euclidean distance between samples indicated in the heatmap color

100 key. C) Volcano plot showing significance versus fold-change. The log fold change in expression is 101 indicated on the $\mathrm{x}$-axis and significance $-\log _{10}(\mathrm{p}$-value $)$ is indicated on the $\mathrm{y}$-axis. DEGs are indicated by 102 red color. D) Heatmap of enriched terms (Canonical Pathways, GO Biological Processes, Hallmark Gene 103 Sets, KEGG Pathway) for DEGs between Qs and NBFs (generated using Metascape). Significance of 
enrichment is indicated on the $\mathrm{x}$-axis in $-\log _{10}(\mathrm{p}$-value). The color code on the $\mathrm{y}$-axis is used to show the clustering and relation of these networks (shown in Supplementary Figure 2E). Detailed list of enriched terms can be found at Additional file 3. E) Network of enriched terms (Supplementary Figure 2D) colored by cluster ID, indicating the relationship between the different enriched terms (cluster ID color correspond to the color code shown in Supplementary Figure 2D y-axis). Nodes that share the same cluster are close to each other. Each circle node represents a term and the size of the circle is proportional to the number of genes that fall into that term, and the identity of the cluster is indicated by its color

111 (nodes of the same color belong to the same cluster). Similar terms are linked by an edge (the thickness of 112 the edge represents the similarity score). One term from each cluster is selected to have its term 113 description shown as label. E) Enriched biological process terms for DEGs that are common in the comparison between Qs vs NBFs generated using Enrichr. The length of the bar represents the significance of that specific gene-set or term, and the color intensity provides additional information about the significance (the brighter the color, the more significant that term is). Statistical information used to generate the graph including the p-value and other enriched terms are provided in Additional file NBFs generated using Enrichr. The length of the bar represents the significance of that specific gene-set or term, and the color intensity provides additional information about the significance (the brighter the color, the more significant that term is). Statistical information used to generate the graph including the pvalue and other enriched terms are provided in Additional file 5.

plot of testis size to body weight. The size of the circles in the scatter plot are proportional to testis to 126 body weight ratio. BMs are depicted in blue and NBMs in black. B) Principal component analysis (PCA) 127 plot showing the clustering of BMs and NBMs based global brain gene expression profile. C) Cluster 128 heatmap of BMs and NBMs brain gene expression. Sample distance was calculated euclidean distance 129 matrix computation and cluster agglomeration was done using ward.D2 method. Heatmap colors indicate 
the euclidean distance between samples indicated in the heatmap color key. D) Volcano plot showing the significance versus fold-change. The log fold change in expression in indicated on the $\mathrm{x}$-axis and significance in $-\log _{10}(\mathrm{p}$-value $)$ is indicated on the y-axis. DEGs are indicated by the red color. E) Heatmap of enriched terms (Canonical Pathways, GO Biological Processes, Hallmark Gene Sets, KEGG Pathway) for DEGs between BMs and NBMs (generated using Metascape). Significance of enrichment is indicated on the $\mathrm{x}$-axis in $-\log _{10}(\mathrm{p}$-value). The color code on the $\mathrm{y}$-axis is used to show the clustering and relation of this networks (shown in Supplementary Figure 3F). Detailed list of enriched terms can be found at Additional file 7. F) Network of enriched terms (Supplementary Figure 3E) colored by cluster ID, indicating the relationship between the different enriched terms (cluster ID color correspond to the color code shown in Supplementary Figure $3 E$ y-axis). Nodes that share the same cluster are close to each that fall into that term, and the identity of the cluster is indicated by its color (nodes of the same color belong to the same cluster). Similar terms are linked by an edge (the thickness of the edge represents the similarity score). One term from each cluster is selected to have its term description shown as label. G)

144 Enriched biological process terms for DEGs that are common in the comparison between BMs vs NBMs generated using Enrichr. The length of the bar represents the significance of that specific gene-set or term, and the color intensity provides additional information about the significance (the brighter the color, the

147 more significant that term is). Statistical information used to generate the graph including the p-value and other enriched terms are provided in Additional file 8. H) Enriched molecular functions terms in for DEGs that are common in the comparison between BMs vs NBMs generated using Enrichr. The length of the bar represents the significance of that specific gene-set or term, and the color intensity provides

151 additional information about the significance (the brighter the color, the more significant that term is).

152 Statistical information used to generate the graph including the p-value and other enriched terms are 153 provided in Additional file 9. 


\section{Supplementary Figure 4: Gene expression differences between the brains of Qs and BMs. A)}

Principal component analysis (PCA) plot showing the clustering of Qs and BMs based global brain gene calculated euclidean distance matrix computation and cluster agglomeration was done using ward.D2 method. Heatmap color indicates the euclidean distance between samples indicated in the heatmap color key. C) Volcano plot showing significance versus fold-change. The log fold change in expression is indicated on the $\mathrm{x}$-axis and significance in $-\log _{10}(\mathrm{p}$-value $)$ is indicated on the $\mathrm{y}$-axis. DEGs are indicted by clustering and relation of this networks (shown in Supplementary Figure 4E). Detailed list of enriched terms can be found at Additional file 11. E) Network of enriched terms (Supplementary Figure 4D) colored by cluster ID, indicating the relationship between the different enriched terms (cluster ID color correspond to the color code shown in Supplementary Figure 4D y-axis). Nodes that share the same 
more significant that term is). Statistical information used to generate the graph including the p-value and other enriched terms are provided in Additional file 13.

\section{Supplementary Figure 5: Gene expression differences between the brains of NBFs and NBMs. A)}

Cluster heatmap of NBFs and NBMs gene brain gene expression. Sample distance was calculated euclidean distance matrix computation and cluster agglomeration was done using ward.D2 method. Heatmap color indicate the euclidean distance between samples indicated in the heatmap color key. B) Volcano plot showing the significance versus fold-change. The log fold change in expression in indicated on the $\mathrm{x}$-axis and significance in $-\log _{10}(\mathrm{p}$-value $)$ is indicated on the $\mathrm{y}$-axis. DEGs are indicated by the red color.

Supplementary Figure 6: Venn diagrams showing the number of DEGs in the brain that were expression in the comparison between BMs vs NBFs and BMs vs NBMs. H) Venn diagram showing DEGs that also show lower expression in the comparison between BMs vs NBFs and BMs vs NBMs. ovary samples. Sample distance was calculated euclidean distance matrix computation and cluster 
agglomeration was done using ward.D2 method. Heatmap color indicates the euclidean distance between samples indicated in the heatmap color key. B) Volcano plot showing the significance versus fold-change. The $\log$ fold change in expression in indicated on the $\mathrm{x}$-axis and significance in $-\log _{10}(\mathrm{p}$-value $)$ is indicated on the y-axis. DEGs are indicated by the red color. C) Network of enriched terms colored by cluster ID, indicating the relationship between the different enriched terms (Figure 3D). Nodes that share the same cluster are close to each other. Each circle node represents a term and the size of the circle is proportional to the number of genes that fall into that term, and the identity of the cluster is indicated by

214 its color (nodes of the same color belong to the same cluster). Similar terms are linked by an edge (the 215 thickness of the edge represents the similarity score). One term from each cluster is selected to have its 216 term description shown as label. D) Enriched biological processes for DEGs between Qs vs NBFs ovary generated using Enrichr. The length of the bar represents the significance of that specific gene-set or term, and the color intensity provides additional information about the significance (the brighter the color, the more significant that term is). Statistical information used to generate the graph including the p-value and other enriched terms are provided in Additional file 27a. E) Enriched molecular functions for DEGs that specific gene-set or term, and the color intensity provides additional information about the significance (the brighter the color, the more significant that term is). Statistical information used to generate the graph including the p-value and other enriched terms are provided in Additional file $28 \mathrm{~A}$. KEGG Pathway) for DEGs that show lower expression in Q ovary (generated using Metascape). The color of the bar graph is proportion to the p-values. Significance of enrichment is indicated on the $\mathrm{x}$-axis in $-\log _{10}(p$-value $)$. The color code on the y-axis is used to show the clustering and relation of these networks (shown in Supplementary Figure 8B). More information about the enriched terms can be found in Additional file 26B. B) Network of enriched terms colored by cluster ID, indicating the relationship 
between the different enriched terms (Supplementary Figure 8A). Nodes that share the same cluster are close to each other. Each circle node represents a term and the size of the circle is proportional to the number of genes that fall into that term, and the identity of the cluster is indicated by its color (nodes of the same color belong to the same cluster). Similar terms are linked by an edge (the thickness of the edge represents the similarity score). One term from each cluster is selected to have its term description shown as label. C) Enriched biological processes for DEGs between Qs and NBFs ovary and also show lower expression in the Qs generated using Enrichr. The length of the bar represents the significance of that specific gene-set or term, and the color intensity provides additional information about the significance (the brighter the color, the more significant that term is). Statistical information used to generate the graph including the p-value and other enriched terms are provided in Additional file 27B. D) Enriched molecular functions for DEGs between Qs and NBFs ovary and also show lower expression in the Qs generated using Enrichr. The length of the bar represents the significance of that specific gene-set or term, and the color intensity provides additional information about the significance (the brighter the color, the more significant that term is). Statistical information used to generate the graph including the p-value and other enriched terms are provided in Additional file $28 B$.

A) Heatmap of enriched terms (Canonical Pathways, GO Biological Processes, Hallmark Gene Sets, KEGG Pathway) for DEGs that show higher expression in Qs ovary (generated using Metascape). The color of the bar graph is proportion to the p-values. Significance of enrichment is indicated on the $\mathrm{x}$ axis in $-\log _{10}$ (p-value). The color code on the $y$-axis is used to show the clustering and relation of this networks (shown in Supplementary Figure 9B). More information about the enriched terms can be found in Additional file 26C. B) Network of enriched terms colored by cluster ID, indicating the relationship between the different enriched terms (Supplementary Figure 9A). Nodes that share the same cluster are

257 close to each other. Each circle node represents a term and the size of the circle is proportional to the 258 number of genes that fall into that term, and the identity of the cluster is indicated by its color (nodes of 
the same color belong to the same cluster). Similar terms are linked by an edge (the thickness of the edge represents the similarity score). One term from each cluster is selected to have its term description shown as label. C) Enriched biological processes for DEGs between Qs and NBFs ovary and also show higher expression in the Qs generated using Enrichr. The length of the bar represents the significance of that specific gene-set or term, and the color intensity provides additional information about the significance (the brighter the color, the more significant that term is). Statistical information used to generate the graph including the p-value and other enriched terms are provided in Additional file 27C. D) Enriched molecular functions for DEGs between Qs and NBFs ovary and also show higher expression in the Q generated using Enrichr. The length of the bar represents the significance of that specific gene-set or term, and the color intensity provides additional information about the significance (the brighter the color, the more significant that term is). Statistical information used to generate the graph including the p-value and other enriched terms are provided in Additional file $28 C$. magnification) through the ovary of non-breeding (A) and Queen (B) NMRs: S stroma, O oocyte, P

274 primordial follicle, $1^{\circ}$ primary follicle, $2^{\circ}$ secondary follicle, $2^{\circ *}$ secondary follicle with two oocytes, $3^{\circ}$ 275 tertiary follicle. oogenesis/folliculogenesis. Mouse gene cluster that show a decrease in expression from the primary to small antral stage (cluster 1) and another gene cluster (cluster 5: where gene expression increased at small antral and large antral follicle follicle stages) were obtained from (50) (753 genes in total in the two clusters). NMR Qs and NBFs ovary expression level was mapped to these mouse folliculogenesis genes 
was plotted (x-axis: clusters, y-axis: expression level in $\log _{2}$ ). B) Violin plot in combination with boxplot showing the expression level of Qs and NBFs genes (NMR ovary) for DEGs that show up and down regulation in mouse infant vs adult whole ovary comparison (51). From 7021 DEGs that show up or down regulation in mouse infant vs adult whole ovary gene expression comparison (51) 4494 genes that show $>2$ fold change (up or down) were extracted, and their expression in Qs and NBFs (NMR ovary) was plotted. x-axis: differential expression group (down, DEGs that show lower expression in adult mouse ovary compared to infant; and up, for genes that show higher expression in adult mouse ovary compared to infant); y-axis expression level in $\log _{2}$ scale. C) Bar plot showing the expression level of mouse oocyte specific genes in NMR Qs and NBFs ovary RNA-seq data. Mouse oocyte specific genes were obtained from [ref], and their average expression level in Q and NBF NMR ovaries (RNA-seq data) was plotted (xaxis: gene name, y-axis: expression level in $\log _{2}$ scale). D) Expression of Cyp19al (Aromatase) in Q and NBF NMR ovaries (x-axis, sample name; y-axis, expression level in counts per million (CPM)).

DEGs. A) Cluster heatmap of testis samples. Sample distance was calculated euclidean distance matrix computation and cluster agglomeration was done using ward.D2 method. Heatmap colors indicate the euclidean distance between samples shown in the heatmap color key. B) Volcano plot showing the significance versus fold-change. The log fold change in expression in indicated on the $x$-axis and significance in $-\log _{10}(\mathrm{p}$-value $)$ is indicated on the y-axis. DEGs are indicated by the red color. C) Network of enriched terms colored by cluster ID, indicating the relationship between the different enriched terms (Figure $4 F$ ). Nodes that share the same cluster are close to each other. Each circle node represents a term and the size of the circle is proportional to the number of genes that fall into that term, and the identity of the cluster is indicated by its color (nodes of the same color belong to the same cluster). Similar terms are

307 linked by an edge (the thickness of the edge represents the similarity score). One term from each cluster is

308 selected to have its term description shown as label. D) Enriched biological processes (generated using 309 Enrichr). The length of the bar represents the significance of that specific gene-set or term, and the color 310 intensity provides additional information about the significance (the brighter the color, the more 
311 significant that term is). Statistical information used to generate the graph including the p-value and other

312 enriched terms are provided in Additional file 31A. E) Enriched molecular functions (generated using

313 Enrichr). The length of the bar represents the significance of that specific gene-set or term, and the color

314 intensity provides additional information about the significance (the brighter the color, the more

315 significant that term is). Statistical information used to generate the graph including the p-value and other

316 enriched terms are provided in Additional file $32 \mathrm{~A}$.

Supplementary Figure 13: Gene enrichment for DEGs that also show lower expression in BM

testes. A) Heatmap of enriched terms (Canonical Pathways, GO Biological Processes, Hallmark Gene

Sets, KEGG Pathway) for DEGs that show lower expression in BMs (generated using Metascape). The

color code on the y-axis is used to show the clustering and relation of enriched networks (Supplementary

Figure 13B). Detailed list of enriched terms and genes that belong to these terms is provided in Additional

file $30 B$. B) Network of enriched terms found in above colored by cluster ID, indicating the relationship between the different enriched terms (Supplementary Figure 13A). Gene enrichment was generated using DEGs that show higher expression in BMs compared to NBMs. Nodes that share the same cluster are close to each other. Nodes that share the same cluster are close to each other. Each term is represented by a circle node, where its size is proportional to the number of input genes fall into that term, and its color represent its cluster identity (i.e., nodes of the same color belong to the same cluster). Similar terms are linked by an edge (the thickness of the edge represents the similarity score). One term from each cluster is selected to have its term description shown as label. C) Enriched biological processes (generated using Enrichr). The length of the bar represents the significance of that specific gene-set or term, and the color intensity provides additional information about the significance (the brighter the color, the more significant that term is). Statistical information used to generate the graph including the p-value and other enriched terms are provided in Additional file 31B. D) Enriched molecular functions (generated using Enrichr). The length of the bar represents the significance of that specific gene-set or term, and the color intensity provides additional information about the significance (the brighter the color, the more 
significant that term is). Statistical information used to generate the graph including the p-value and other enriched terms are provided in Additional file $32 B$.

\section{Supplementary Figure 14: Gene enrichment for DEGs that also show higher expression in breeding}

male testes. A) Heatmap of enriched terms (Canonical Pathways, GO Biological Processes, Hallmark

Gene Sets, KEGG Pathway) for DEGs that show higher expression in BMs (generated using Metascape).

343 Enriched terms significance is colored by p-values. The color code on the y-axis is used to show the

344 clustering and relation of enriched networks (Supplementary Figure 14B). Detailed list of enriched terms

345 and genes that belong to these terms is provided in Additional file 30C. B) Network of enriched terms

346 found in above colored by cluster ID, indicating the relationship between the different enriched terms

347 (Supplementary Figure 14A). Gene enrichment was generated using genes that show higher expression in

348 breeding males compared to NBMs. Nodes that share the same cluster are close to each other. Nodes that

349 share the same cluster are close to each other. Each term is represented by a circle node, where its size is

350 proportional to the number of input genes fall into that term, and its color represent its cluster identity

351 (i.e., nodes of the same color belong to the same cluster). Similar terms are linked by an edge (the

352 thickness of the edge represents the similarity score). One term from each cluster is selected to have its

353 term description shown as label. C) Enriched biological processes. The length of the bar represents the

354 significance of that specific gene-set or term, and the color intensity provides additional information

355 about the significance (the brighter the color, the more significant that term is). Statistical information

356 used to generate the graph including the p-value and other enriched terms are provided in Additional file

357 31C. D) Enriched molecular functions. The length of the bar represents the significance of that specific

358 gene-set or term, and the color intensity provides additional information about the significance (the

359 brighter the color, the more significant that term is). Statistical information used to generate the graph

360 including the p-value and other enriched terms are provided in Additional file $32 \mathrm{C}$. 
Supplementary Figure 15: NMR BM and NBM testis histology. Representative sections through the testes of adult (A,C) breeding and (B,D) non-breeding male NMRs showing spermatogenesis; (A) and (B) are higher magnification showing examples of the cell types at different stages: $\mathrm{S}_{\mathrm{A}}$ spermatogonia

cell $\left(S_{2}\right.$ secondary spermatocytes not identified). Sections $(\mathbf{C})$ and $(\mathbf{D})$ are lower magnification (from the respective individuals) and show the marked differences in the relative amount of interstitial tissue (mainly Leydig cells) to seminiferous tubules (bounded by the dotted lines).

Supplementary Figure 16: A) Potentially dopaminergic neuron cell groups (A8-A16), expressing the enzyme tyrosine hydroxylase but not the dopamine beta-hydroxylase, with their principal projections in the adult rodent brain (adapted from (45)). B) and C) A model describing the possible pathways though which a dopamine could regulate reproductive division of labor; B) in breeding animals (Qs and BMs), increased dopamine production in the hypothalamus suppresses the production of prolactin in from the pituitary. In the absence (or low amounts) of prolactin, GnRH is released from the hypothalamus, which acts on anterior pituitary to facilitate the release of FSH and LH. FSH and LH act on the gonads of

377 breeding animals (Qs and BMs), to bring about normal gonadal development, gametogenesis and the release of sex hormones (estrogen and testosterone). C) in non-breeding naked mole-rats animals, lower levels of dopamine release result in increased production of prolactin by the anterior pituitary, which in turn suppresses the release of GnRH from the hypothalamus. In the absence of normal GnRH secretion, the anterior pituitary produces low levels of FSH and LH, resulting in suppression of normal gonadal development, gametogenesis and the release of sex hormones. D) A model showing the possible arrest point for NBF NMRs (indicated by the color graduation in the horizontal bar). The Q ovary can complete all the stages of oogesisis, however NBF fail at the stage where they produce estrogen (and are prepubertal in appearance). E) A model showing the possible arrest point where defects in spermatogenesis occur in non-breeding NMRs (indicated by the color graduation in the horizontal bar). Breeding males 
bioRxiv preprint doi: https://doi.org/10.1101/209932; this version posted October 27, 2017. The copyright holder for this preprint (which was not certified by peer review) is the author/funder, who has granted bioRxiv a license to display the preprint in perpetuity. It is made available under aCC-BY-NC-ND 4.0 International license.

387 have the ability to complete all stages of spermatogenesis; however, non-breeding animals fail at post388 meiotic stages and have further deficiencies in sperm maturation. 
bioRxiv preprint doi: https://doi.org/10.1101/209932; this version posted October 27, 2017. The copyright holder for this preprint (which was not certified by peer review) is the author/funder, who has granted bioRxiv a license to display the preprint in perpetuity. It is made available under Additional file legends

Additional file 1: Animals/samples used and details of their subsequent analysis.

Tabs in the table indicate:

A) Sacrificed animals (NMRs) for the experiments: Table headers indicate animal/sample number in colony, social group (colony) where the animal belonged, year of birth, age (in years), body weight (in gram), whether used for sequencing (RNA-seq) or Immunofluorescene (IF), and the name used in the manuscript.

B) RNA-seq information. Table headers indicate: Tissue, name used to identify sample in manuscript (Name in manuscript), RNA-seq sample number, total sequence obtained (number of reads), length of each read in bp (sequence length), together with percentage GC content of total number of reads.

Additional file 2: DEGs between brains of Qs and NBFs.

Tabs in the table indicate:

All DEGs Qs vs NBFs: all DEGs between brains of Qs and NBFs.

DEG up in $Q$ (Qs vs $N B F s)$ : DEGs with higher expression in Qs brains.

DEG down in Qs (Qs vs NBFs): DEGs with lower expression in Qs brains.

In all tabs the table headers indicate: the naked mole rat gene id from our transcript assembly (NMR_geneID); the $\log$ fold change $(\log \mathrm{FC})$; counts per million in $\log$ scale $(\log \mathrm{CPM})$; pvalue (PValue); false discovery rate using Benjamini-Hochberg (FDR); annotation of NMR_geneID to mouse Ensembl gene ID (Ensembl_geneID); and annotation of NMR_geneID to mouse gene name (gene_name).

Additional file 3: Result of enrichment analysis on DEGs between brains of Qs and NBFs using Metascape.

The GroupID, ID for clustering of related terms, the enriched terms (Term), the description of the enriched terms (Description), the p-value in $\log 10$ scale (p-values calculated based on 
bioRxiv preprint doi: https://doi.org/10.1101/209932; this version posted October 27, 2017. The copyright holder for this preprint (which was not certified by peer review) is the author/funder, who has granted bioRxiv a license to display the preprint in perpetuity. It is made available under accumulative hypergeometric destriBution), using the Banjamini-Hochberg procedure to account for multiple testing), list of Symbols of upload hits in this term (Symbols).

Additional file 4: Result of enrichment analysis on DEGs between brains of Qs and NBFs using Enrichr (GO Biological Process).

Enrichment analysis was performed as described in material and methods. The table headers indicate: Term; enriched GO Biological Process; p-value, computed by Fisher's exact test; adjusted p-value, an adjusted p-value using the Benjamini-Hochberg method for correction for multiple hypotheses testing; the rank score or (z-score), computed using a modification to Fisher's exact test in which a z-score was computed for deviation from an expected rank; combined score, a combination of the p-value and z-score calculated by multiplying the two scores $(\mathrm{c}=\log (\mathrm{p}) * \mathrm{z})$; Genes, belonging to the enriched category.

Additional file 5: Result of enrichment analysis on DEGs between brains of Qs and NBFs using Enrichr (GO molecular functions).

The table headers indicate: Term; enriched GO molecular functions; p-value, computed by fisher's exact test; adjusted p-value, an adjusted p-value using the Benjamini-Hochberg method for correction for multiple hypotheses testing; the rank score or (z-score), computed using a modification to Fisher's exact test in which a z-score was computed for deviation from an expected rank; combined score, a combination of the p-value and z-score calculated by multiplying the two scores $(\mathrm{c}=\log (\mathrm{p}) * \mathrm{z})$; Genes, belonging to the enriched category.

Additional file 6: DEGs between brains of BMs and NBMs.

Tabs in the table indicate:

All_DEGs (BMs vs NBMs) tab: all DEGs between brains of BMs vs NBMs.

DEG_up_in_BMs (BMs vs NBMs) tab: DEGs with higher expression in BMs brains.

DEG_down_in_BMs (BMs vs NBMs) tab: DEGs with lower expression in BMs brains. 
bioRxiv preprint doi: https://doi.org/10.1101/209932; this version posted October 27, 2017. The copyright holder for this preprint (which was not certified by peer review) is the author/funder, who has granted bioRxiv a license to display the preprint in perpetuity. It is made available under

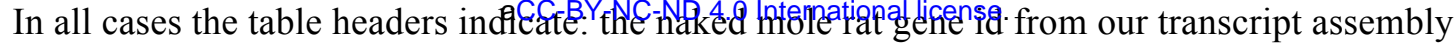

(NMR_geneID); the $\log$ fold change $(\log \mathrm{FC})$; counts per million in $\log$ scale $(\log \mathrm{CPM})$;

pvalue (PValue); false discovery rate using Benjamini-Hochberg (FDR); annotation of NMR_geneID to mouse Ensembl gene ID (Ensembl_geneID); and annotation of NMR_geneID to mouse gene name (gene_name).

Additional file 7: Result of enrichment analysis on DEGs between brains of BMs and NBMs using Metascape.

The table headers indicate: the GroupID, ID for clustering of related terms, the enriched terms (Term), the description of the enriched terms (Description), the p-value in $\log 10$ scale (pvalues calculated based on accumulative hypergeometric distribution), q-value in $\log 10$ scale (q-values are calculated using the Banjamini-Hochberg procedure to account for multiple testing), list of Symbols of upload hits in this term (Symbols).

Additional file 8: Result of enrichment analysis on DEGs between brains of BMs and NBMs using Enrichr (GO Biological Process).

Enrichment analysis was performed as described in material and methods. The table headers indicate: Term; enriched GO Biological Process; p-value, computed by fisher's exact test; adjusted P-value, an adjusted p-value using the Benjamini-Hochberg method for correction for multiple hypotheses testing; the rank score or (z-score), computed using a modification to Fisher's exact test in which a z-score was computed for deviation from an expected rank; combined score, a combination of the p-value and z-score calculated by multiplying the two scores $(\mathrm{c}=\log (\mathrm{p}) * \mathrm{z})$; Genes, belonging to the enriched category.

Additional file 9: Result of enrichment analysis on DEGs between brains of BMs and NBMs using Enrichr (GO molecular functions).

The table headers indicate: Term; enriched GO molecular functions; p-value, computed by fisher's exact test; adjusted p-value, an adjusted p-value using the Benjamini-Hochberg 
bioRxiv preprint doi: https://doi.org/10.1101/209932; this version posted October 27, 2017. The copyright holder for this preprint (which was not certified by peer review) is the author/funder, who has granted bioRxiv a license to display the preprint in perpetuity. It is made available under method for correction for multipłe hypotheses testingtioftil lifanke score or (z-score), computed using a modification to Fisher's exact test in which a z-score was computed for deviation from an expected rank; combined score, a combination of the p-value and z-score calculated by multiplying the two scores $(\mathrm{c}=\log (\mathrm{p}) * \mathrm{z})$; Genes, belonging to the enriched category.

Additional file 10: DEGs between brains of Qs and BMs.

Tabs in the table indicate:

All_DEGs (Qs_vs_BMs) tab: all DEGs between brains of Qs and BMs.

DEG_and_up_in_Q (Qs_vs_BMs) tab: DEGs with higher expression in Qs brain. DEG_and_down_in_Q (Qs_vs_BMs) tab: DEGs with lower expression in Qs brain.

In all cases, the table headers indicate: the naked mole rat gene id from our transcript assembly (NMR_geneID); the log fold change $(\log \mathrm{FC})$; counts per million in $\log$ scale $(\log \mathrm{CPM}) ;$ p-value (PValue); false discovery rate using Benjamini-Hochberg (FDR); annotation of NMR_geneID to mouse Ensembl gene ID (Ensembl_geneID); and annotation of NMR_geneID to mouse gene name (gene_name).

Additional file 11: Result of enrichment analysis on DEGs between brains of Qs and BMs using Metascape.

The table headers indicate: the GroupID, ID for clustering of related terms, the enriched terms (Term), the description of the enriched terms (Description), the p-value in $\log 10$ scale (pvalues calculated based on accumulative hypergeometric distribution), q-value in $\log 10$ scale (q-values are calculated using the Banjamini-Hochberg procedure to account for multiple testing), list of Symbols of upload hits in this term (Symbols).

Additional file 12: Result of enrichment analysis on DEGs between brains of Qs and BMs using Enrichr (GO Biological Process). 
bioRxiv preprint doi: https://doi.org/10.1101/209932; this version posted October 27, 2017. The copyright holder for this preprint (which was not certified by peer review) is the author/funder, who has granted bioRxiv a license to display the preprint in perpetuity. It is made available under The table headers indicate: Term, fisher's exact test; adjusted p-value, an adjusted p-value using the Benjamini-Hochberg method for correction for multiple hypotheses testing; the rank score or (z-score), computed using a modification to Fisher's exact test in which a z-score was computed for deviation from an expected rank; combined score, a combination of the p-value and z-score calculated by multiplying the two scores $(\mathrm{c}=\log (\mathrm{p}) * \mathrm{z})$; Genes, belonging to the enriched category.

Additional file 13: Result of enrichment analysis on DEGs between brains of Qs and BMs using Enrichr (GO molecular functions).

The table headers indicate: Term; enriched GO molecular functions; p-value, computed by fisher's exact test; adjusted p-value, an adjusted p-value using the Benjamini-Hochberg method for correction for multiple hypotheses testing; the rank score or (z-score), computed using a modification to Fisher's exact test in which a z-score was computed for deviation from an expected rank; combined score, a combination of the p-value and z-score calculated by multiplying the two scores $(c=\log (p) * z)$; Genes, belonging to the enriched category.

Additional file 14: DEGs between brains of NBFs and NBMs.

Tabs in the table indicate:

All DEGs (NBFs vs NBMs) tab: all DEGs brains of NBFs and NBMs.

DEGs up in NBF (NBFs vs NBMs) tab: DEGs with higher expression in NBFs brain.

DEGs down in NBF (NBFs vs NBMs) tab: DEGs with lower expression in NBFs brain.

In all tabs the table headers indicate: the naked mole rat gene id from our transcript assembly (NMR_geneID); the $\log$ fold change $(\log \mathrm{FC})$; counts per million in $\log$ scale $(\log \mathrm{CPM})$; $\mathrm{p}$ value (PValue); false discovery rate using Benjamini-Hochberg (FDR); annotation of NMR geneID to mouse Ensembl gene ID (Ensembl geneID); and annotation of NMR_geneID to mouse gene name (gene_name). 
bioRxiv preprint doi: https://doi.org/10.1101/209932; this version posted October 27, 2017. The copyright holder for this preprint (which was not certified by peer review) is the author/funder, who has granted bioRxiv a license to display the preprint in perpetuity. It is made available under

Additional file 15: DEGs betweeffbring

Tabs in the table indicate:

All_DEGs (Qs_vs_NBMs) tab: all DEGs between brains of Qs and NBMs.

DEG_up_in_Q (Qs_vs_NBMs) tab: DEGs with higher expression in Qs brain.

DEG_down_in_Q (Qs_vs_NBMs) tab: DEGs with lower expression in Qs brain.

In all cases the table headers indicate: the naked mole rat gene id from our transcript assembly (NMR_geneID); the log fold change ( $\log \mathrm{FC})$; counts per million in $\log$ scale $(\log \mathrm{CPM})$; pvalue (PValue); false discovery rate using Benjamini-Hochberg (FDR); annotation of NMR_geneID to mouse Ensembl gene ID (Ensembl_geneID); and annotation of NMR_geneID to mouse gene name (gene_name).

Additional file 16: Defining queen specific genes (Q genes). The list of DEGs in the comparison between Qs vs NBFs, Qs vs NBMs, Qs vs BMs were mapped to each other to identify queen specific genes.

The different tables in the excel sheet are:

Q vs NBF NBM BM all tab: DEGs between Qs vs NBFs, Qs vs NBMs and Qs vs BMs.

$\boldsymbol{Q}$ vs $\boldsymbol{N B F}$ NBM all tab: all DEGs between Qs vs NBFs and Qs vs NBMs.

$Q$ vs NBF NBM BM down in $Q$ tab: genes that are differentially expressed in the comparison (Qs vs NBFs, Qs vs NBMs, and Qs vs BMs) and also show lower expression in Q (down regulation in Q)

$Q$ vs NBF NBM down in $Q$ tab: genes that are differentially expressed in the comparison (Qs vs NBFs, Qs vs NBMs) and also show lower expression in Q (down regulation in Q)

$Q$ vs $N B F$ NBM BM up in $Q$ tab: genes that are differentially expressed in the comparison (Qs vs NBFs, Qs vs NBMs, and Qs vs BMs) and also show higher expression in Q (up regulation in Q)

$Q$ vs NBF NBM up in $Q$ tab: genes that are differentially expressed in the comparison (Qs vs NBFs, Qs vs NBMs) and also show higher expression in Q (up regulation in Q) 
bioRxiv preprint doi: https://doi.org/10.1101/209932; this version posted October 27, 2017. The copyright holder for this preprint (which was not certified by peer review) is the author/funder, who has granted bioRxiv a license to display the preprint in perpetuity. It is made available under

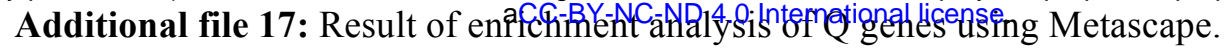

The table headers indicate: GroupID, ID for clustering of related terms, the enriched terms (Term), the description of the enriched terms (Description), the p-value in $\log 10$ scale (pvalues calculated based on accumulative hypergeometric distribution), q value in $\log 10$ scale (q-values are calculated using the Banjamini-Hochberg procedure to account for multiple testing), list of Symbols of upload hits in this term (Symbols).

Additional file 18: Result of enrichment analysis of Q genes using Enrichr (GO Biological Process).

The table headers indicate: the Term; enriched GO Biological Process; P-value, computed by fisher's exact test; adjusted p-value, an adjusted p-value using the Benjamini-Hochberg method for correction for multiple hypotheses testing; the rank score or (z-score), computed using a modification to Fisher's exact test in which a z-score was computed for deviation from an expected rank; combined score, a combination of the p-value and z-score calculated by multiplying the two scores $(\mathrm{c}=\log (\mathrm{p}) * \mathrm{z})$; Genes, belonging to the enriched category.

Additional file 19: Result of enrichment analysis of Q genes using Enrichr (GO molecular functions).

The table headers indicate: the Term; enriched GO molecular functions; p-value, computed by fisher's exact test; adjusted P-value, an adjusted p-value using the Benjamini-Hochberg method for correction for multiple hypotheses testing; the rank score or (z-score), computed using a modification to Fisher's exact test in which a z-score was computed for deviation from an expected rank; combined score, a combination of the p-value and z-score calculated by multiplying the two scores $(\mathrm{c}=\log (\mathrm{p}) * \mathrm{z})$; Genes, belonging to the enriched category.

Additional file 20: DEGs between brains of BMs and NBFs.

Tabs in the table indicate:

All DEGs BMs vs NBFs tab: all DEGs brains of BMs and NBFs. 
bioRxiv preprint doi: https://doi.org/10.1101/209932; this version posted October 27, 2017. The copyright holder for this preprint (which was not certified by peer review) is the author/funder, who has granted bioRxiv a license to display the preprint in perpetuity. It is made available under

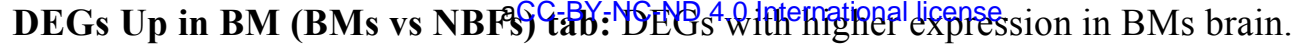

DEGs down in BM (BMs vs NBFs) tab: DEGs with lower expression in BMs brain.

In all cases the table headers indicate: the naked mole rat gene id from our transcript assembly

(NMR_geneID); the $\log$ fold change $(\log \mathrm{FC})$; counts per million in $\log$ scale $(\log \mathrm{CPM})$; p-

value (PValue); false discovery rate using Benjamini-Hochberg (FDR); annotation of

NMR_geneID to mouse Ensembl gene ID (Ensembl_geneID); and annotation of

NMR_geneID to mouse gene name (gene_name).

Additional file 21: Defining BM specific genes (BM genes). The list of DEGs in the comparison between BMs vs NBFs, BMs vs NBMs, were mapped to each other to identify breeding male specific genes.

Additional file 22: Result of enrichment analysis of BM genes using Metascape.

Enrichment analysis was performed as described in material and methods. The output of the enrichment analysis is presented here. The table headers indicate: The GroupID, ID for clustering of related terms, the enriched terms (Term), the description of the enriched terms (Description), the p-value in $\log 10$ scale (p-values calculated based on accumulative hypergeometric distribution), $\mathrm{q}$ value in $\log 10$ scale (q-values are calculated using the Banjamini-Hochberg procedure to account for multiple testing), list of Symbols of upload hits in this term (Symbols).

Additional file 23: Result of enrichment analysis of BM genes using Enrichr (GO Biological Process).

The table headers indicate: the Term; enriched GO Biological Process; p-value, computed by fisher's exact test; adjusted p-value, an adjusted p-value using the Benjamini-Hochberg method for correction for multiple hypotheses testing; the rank score or (z-score), computed using a modification to Fisher's exact test in which a z-score was computed for deviation from 
bioRxiv preprint doi: https://doi.org/10.1101/209932; this version posted October 27, 2017. The copyright holder for this preprint (which was not certified by peer review) is the author/funder, who has granted bioRxiv a license to display the preprint in perpetuity. It is made available under

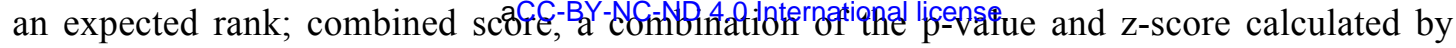
multiplying the two scores $(\mathrm{c}=\log (\mathrm{p}) * \mathrm{z})$; Genes, belonging to the enriched category.

Additional file 24: Result of enrichment analysis of BM genes using Enrichr (GO molecular functions).

The table headers indicate: the Term; enriched GO molecular functions; p-value, computed by fisher's exact test; adjusted p-value, an adjusted p-value using the Benjamini-Hochberg method for correction for multiple hypotheses testing; the rank score or (z-score), computed using a modification to Fisher's exact test in which a z-score was computed for deviation from an expected rank; combined score, a combination of the p-value and z-score calculated by multiplying the two scores $(\mathrm{c}=\log (\mathrm{p}) * \mathrm{z})$; Genes, belonging to the enriched category.

Additional file 25: DEGs between Qs and NBFs ovary.

Tabs in the table indicate:

All_DEGs ( $\left.Q s_{-} v \boldsymbol{v s}_{-} N B F s\right)$ tab: all DEGs between ovaries of Qs and NBFs.

DEG_and_down_in_Q (Qs_vs_NBFs) tab: DEGs with lower expression in Qs ovary.

$\boldsymbol{D E G}$ _and_up_in_Q (Qs_vs_NBFs) tab: DEGs with lower expression in in Qs ovary.

In all tabs the table headers indicate: the naked mole rat gene id from our transcript assembly (NMR_geneID); the $\log$ fold change $(\log \mathrm{FC})$; counts per million in $\log$ scale $(\log \mathrm{CPM})$; pvalue (PValue); false discovery rate using Benjamini-Hochberg (FDR); annotation of NMR_geneID to mouse Ensembl gene ID (Ensembl_geneID); and annotation of NMR_geneID to mouse gene name (gene_name).

Additional file 26: Result of enrichment analysis on DEGs between Qs and NBFs ovary using Metascape. The table headers indicate: The GroupID, ID for clustering of related terms, the enriched terms (Term), the description of the enriched terms (Description), the p-value in $\log 10$ scale (p-values calculated based on accumulative hypergeometric distribution), q value 
bioRxiv preprint doi: https://doi.org/10.1101/209932; this version posted October 27, 2017. The copyright holder for this preprint (which was not certified by peer review) is the author/funder, who has granted bioRxiv a license to display the preprint in perpetuity. It is made available under

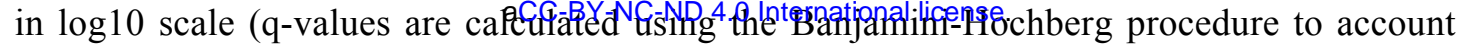
for multiple testing), list of symbols of upload hits in this term (Symbols).

The tabs in the table show:

A) all DEGs: enriched pathways, Biological Process, and molecular functions for all DEGs between Qs and NBFs ovary.

B) DEGs up in Q: enriched pathways, Biological Process, and molecular functions for DEGs between Qs and NBFs ovary, and up regulated in Q compared to NBF.

C) DEGs down in Q: enriched pathways, Biological Process, and molecular functions for DEGs between Qs and NBFs ovary, and down regulated in Q compared to NBF.

Additional file 27: Result of enrichment analysis on DEGs between Qs and NBFs ovary using Enrichr (GO Biological Process).

The table headers indicate: the Term; enriched GO Biological Process; p-value, computed by fisher's exact test; adjusted p-value, an adjusted p-value using the Benjamini-Hochberg method for correction for multiple hypotheses testing; the rank score or (z-score), computed using a modification to Fisher's exact test in which a z-score was computed for deviation from an expected rank; combined score, a combination of the p-value and z-score calculated by multiplying the two scores $(\mathrm{c}=\log (\mathrm{p}) * \mathrm{z})$; Genes, belonging to the enriched category. The tabs in the table show:

A) All DEGs: enriched Biological Process for all DEGs between Qs and NBFs ovary.

B) DEGs down in Q: enriched Biological Process for DEGs between Q and NBFs ovary, and down regulated in Qs compared to NBFs.

C) DEGs up in Q: enriched Biological Process for DEGs between Qs and NBFs ovary, and up regulated in Qs compared to NBFs.

Additional file 28: Result of enrichment analysis on DEGs between Qs and NBFs ovary using Enrichr (GO molecular functions). 
bioRxiv preprint doi: https://doi.org/10.1101/209932; this version posted October 27, 2017. The copyright holder for this preprint (which was not certified by peer review) is the author/funder, who has granted bioRxiv a license to display the preprint in perpetuity. It is made available under

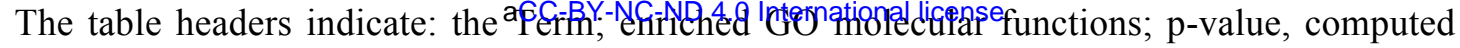

by fisher's exact test; adjusted p-value, an adjusted p-value using the Benjamini-Hochberg method for correction for multiple hypotheses testing; the rank score or (z-score), computed using a modification to Fisher's exact test in which a z-score was computed for deviation from an expected rank; combined score, a combination of the p-value and z-score calculated by multiplying the two scores $(\mathrm{c}=\log (\mathrm{p}) * \mathrm{z})$; Genes, belonging to the enriched category.

The tabs in the table show:

A) All DEGs: enriched molecular functions for all DEGs between Qs and NBFs ovary.

B) DEGs down in Qs: enriched molecular functions for DEGs between Qs and NBFs ovary, and down regulated in Qs compared to NBFs.

C) DEGs up in Qs: enriched Biological Process for DEGs between Qs and NBFs ovary, and up regulated in Qs compared to NBFs.

Additional file 29: DEGs between BMs and NBMs testis.

Tabs in the table indicate:

All DEGs (BMs vs NBMs) tab: all DEGs between BMs and NBMs testis.

DEG down in BM (BMs vs NBMs) tab: DEGs and higher expression in BMs testis.

DEG up in BM (BMs vs NBMs) tab: DEGs and lower expression in BMs testis.

Germonline genes: Gene list obtained form Germonline (germonline.org)

In all tabs the table headers indicate: the naked mole rat gene id from our transcript assembly (NMR_geneID); the $\log$ fold change $(\log \mathrm{FC})$; counts per million in $\log$ scale $(\log \mathrm{CPM})$; pvalue (PValue); false discovery rate using Benjamini-Hochberg (FDR); annotation of NMR_geneID to mouse Ensembl gene ID (Ensembl_geneID); and annotation of NMR_geneID to mouse gene name (gene_name).

Additional file 30: Result of enrichment analysis on DEGs between BMs and NBMs testis using Metascape. 
bioRxiv preprint doi: https://doi.org/10.1101/209932; this version posted October 27, 2017. The copyright holder for this preprint (which was not certified by peer review) is the author/funder, who has granted bioRxiv a license to display the preprint in perpetuity. It is made available under

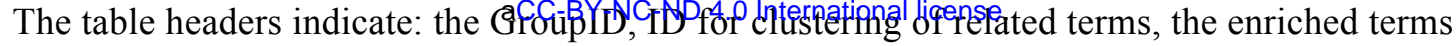

(Term), the description of the enriched terms (Description), the p-value in log10 scale (pvalues calculated based on accumulative hypergeometric distribution), q-value in $\log 10$ scale (q-values are calculated using the Banjamini-Hochberg procedure to account for multiple testing), list of Symbols of upload hits in this term (Symbols).

The tabs in the table show:

A) All DEGs: enriched pathways, Biological Process, and molecular functions for all DEGs between BMs and NBM testis.

B) DEGs down in_BM: enriched pathways, Biological Process, and molecular functions for DEGs between BMs and NBMs testis, and up regulated in BMs compared to NBMs.

C) DEGs up in BM: enriched pathways, Biological Process, and molecular functions for DEGs between BMs and NBMs testis, and up regulated in BMs compared to NBMs.

D) reprod \&steroid respon: selected genes that belong to terms, multicellular organism reproduction, response to steroid hormone, multicellular organism reproduction and response to steroid hormone.

Additional file 31: Result of enrichment analysis on differentially expressed between BMs and NBMs testis using Enrichr (GO Biological Process).

The table headers indicate: Term; enriched GO Biological Process; p-value, computed by fisher's exact test; adjusted p-value, an adjusted p-value using the Benjamini-Hochberg method for correction for multiple hypotheses testing; the rank score or (z-score), computed using a modification to Fisher's exact test in which a z-score was computed for deviation from an expected rank; combined score, a combination of the p-value and z-score calculated by multiplying the two scores $(\mathrm{c}=\log (\mathrm{p}) * \mathrm{z})$; Genes, belonging to the enriched category.

The tabs in the table show:

A) All_deg: GO Biological Process enrichment for all DEGs between BMs and NBMs testis.

B) DEGs up in BM: GO Biological Process enrichment for DEGs between BMs and NBMs testis, and up regulated in BMs compared to NBMs. 
bioRxiv preprint doi: https://doi.org/10.1101/209932; this version posted October 27, 2017. The copyright holder for this preprint (which was not certified by peer review) is the author/funder, who has granted bioRxiv a license to display the preprint in perpetuity. It is made available under

C) DEGs down in BM: GO BG\&IBgic

NBMs testis, and down regulated in BMs compared to NBMs.

Additional file 32: Result of enrichment analysis on DEGs between BMs and NBMs testis using Enrichr (GO molecular functions). The table headers indicate: Term; enriched GO molecular functions; p-value, p-value, computed by fisher's exact test; adjusted p-value, an adjusted p-value using the Benjamini-Hochberg method for correction for multiple hypotheses testing; the rank score or (z-score), computed using a modification to Fisher's exact test in which a z-score was computed for deviation from an expected rank; combined score, a combination of the p-value and z-score calculated by multiplying the two scores $(\mathrm{c}=$ $\log (\mathrm{p}) * \mathrm{z})$; Genes, belonging to the enriched category.

The tabs in the table show:

A) All DEG: GO molecular functions enrichment for DEGs between BMs and NBMs testis.

B) DEG down in BM: GO molecular functions enrichment for DEGs between BMs and NBMs testis, and down regulated in BMs compared to NBMs.

C) DEG up in BM: GO molecular functions enrichment for DEGs between BMs and NBMs testis, and up regulated in BMs compared to NBMs. 